\title{
Momentos de Ruptura y Resolución de la Alianza Terapéutica en el Caso de una Adolescente Diagnosticada con Difusión de Identidad: Su Impacto en el Resultado Terapéutico
}

\section{Moments of Rupture and Resolution of the Therapeutic Alliance in the Case of an Adolescent Diagnosed with Identity Diffusion: Their Impact on Psychotherapeutic Outcomes}

\author{
Nelson Valdés \\ Instituto Milenio para la Investigación en Depresión y Personalidad \\ Diana Gómez \\ Pontificia Universidad Católica de Chile \\ Mahaira Reinel \\ Instituto Milenio para la Investigación en \\ Depresión y Personalidad
}

\begin{abstract}
La alianza terapéutica es un componente importante del proceso terapéutico; sin embargo, pocos estudios han explorado cómo se construye la alianza terapéutica con pacientes adolescentes. Se utilizó un diseño de caso único con una metodología mixta para identificar momentos de ruptura e intentos de resolución de la alianza terapéutica a partir del microanálisis de un caso exitoso de una adolescente con indicadores de una difusión de identidad y sintomatología depresiva. Se le aplicaron el Youth Outcome Questionnaire-Self Report, el Cuestionario de Desarrollo de la Identidad en Adolescentes, el Inventario de Depresión de Beck-I y el Inventario de Resolución de Problemas Revisado. Se analizaron 32 sesiones, utilizando el Sistema de Identificación de Rupturas y Resoluciones de Eubanks, Muran y Safran (2015), identificándose un total de 29 momentos de ruptura (11 de distanciamiento y 18 de confrontación). Se analizó la frecuencia y las características de dichos momentos relevantes, así como su relación con el éxito terapéutico al final del proceso, mediante diferencias pre-post, utilizando Z-ratio. Se discuten las implicancias clínicas de resolver inmediatamente los momentos de ruptura durante la sesión.
\end{abstract}

Palabras clave: rupturas, alianza terapéutica, adolescentes, difusión de identidad, estudio de caso

\begin{abstract}
The therapeutic alliance is an important component of the therapeutic process; however, very few studies have explored how the therapeutic alliance is constructed with adolescent patients. A single case study with a mixed methodology was conducted to identify moments of rupture and attempts to resolve the therapeutic alliance, based on the microanalysis of the successful treatment of an adolescent with identity diffusion indicators and depressive symptomatology. The Youth Outcome Questionnaire-Self Report, the Assessment of Identity Development in Adolescence, the Beck Depression Inventory (BDI-I), and the Social Problem Solving Inventory-Revised were administered. Thirty-two sessions were analyzed using the Ruptures and Resolutions Coding System developed by Eubanks, Muran, and Safran (2015), in which 29 rupture moments were found (11 withdrawals and 18 confrontations). The frequency and characteristics of these relevant moments, as well as their relationship with therapeutic success at the end of the process, were analyzed through pre-post differences, using Z-ratio. The clinical implications of immediately resolving moments of rupture during the session are discussed.
\end{abstract}

Keywords: rupture, therapeutic alliance, adolescents, identity diffusion, case study

La adolescencia es un período de la vida que presenta desafíos, los que, al ser enfrentados de manera adecuada, culminarán en el desarrollo de una identidad integrada, coherente y flexible. Sin embargo, algunos adolescentes experimentan dificultades durante este proceso, debido a predisposiciones biológicas y experiencias de vida. Como resultado, estos adolescentes suelen presentar problemas para autodefinirse,

Nelson Valdés, Investigador Adjunto del Instituto Milenio para la Investigación en Depresión y Personalidad (MIDAP) , Santiago, Chile; Diana Gómez, Programa de Doctorado en Psicoterapia, Escuela de Psicología, Pontificia Universidad Católica de Chile, Santiago, Chile; Mahaira Reinel, Instituto Milenio para la Investigación en Depresión y Personalidad (MIDAP), Santiago, Chile.

La correspondencia relativa a este artículo debe ser dirigida a Nelson Valdés, E-mail: nlvaldes@uc.cl 
viven una dolorosa sensación de incoherencia y vacío crónico, muestran conductas contradictorias, pero sobre todo, una baja tolerancia a la frustración y un bajo control de impulsos. En otras palabras, son adolescentes con serias dificultades para integrar su self de una manera diferenciada, lo que termina constituyendo una patología de la identidad, que consecuentemente será la base para un trastorno de personalidad durante la adultez (Foelsch et al., 2014).

Estas dificultades suponen un déficit en la adaptación al medio, ya que se asocian a conductas disfuncionales de carácter crónico, las que terminan deteriorando las relaciones sociales, familiares y escolares y produciendo un gran sufrimiento. Es justamente la complejidad en las relaciones sociales lo que debe ser considerado al momento de intentar establecer una alianza terapéutica con este tipo de pacientes, ya que repiten ciertos patrones disfuncionales durante la terapia, los que deben ser considerados en la construcción del vínculo para asegurar el éxito terapéutico.

La alianza terapéutica ha sido ampliamente asociada a resultados terapéuticos positivos y a un mayor compromiso del paciente durante la terapia (Bachelor, 2013; Bhola \& Kapur, 2013; Green, 2006; Horvath \& Bedi, 2002; Karver, Handelsman, Fields \& Bickman, 2006; Kazdin \& Whitley, 2006; Liber et al., 2010). Sin embargo, son pocos los estudios realizados con el fin de investigar lo complejo que resulta desarrollar esta alianza durante el proceso terapéutico con pacientes adolescentes (Duncan, Miller, Wampold \& Hubble, 2010; Fernández, Pérez, Gloger \& Krause, 2015; Shirk \& Karver, 2011). A pesar de lo anterior, es un elemento valorado positivamente por los terapeutas, los pacientes y sus padres (Diamond, Siqueland \& Diamond, 2003; Friedlander, Kivlighan Jr. \& Shaffer, 2012; Kazdin \& Whitley, 2006). Considerando estos antecedentes, es relevante facilitar el involucramiento de los adolescentes, de manera que logren sentirse seguros para trabajar terapéuticamente de manera colaborativa (Eyrich-Garg, 2008), teniendo en cuenta las diferencias que existen con respecto al trabajo clínico realizado con adultos (Shirk \& Karver, 2011). Esta premisa resulta fundamental, sobre todo cuando se ha demostrado que la calidad de la relación terapéutica está altamente relacionada con el resultado terapéutico (Martin, Garske \& Davis, 2000) y la adherencia al tratamiento (Bolton Oetzel \& Scherer, 2003; Lindsey et al., 2014).

Existen distintas maneras de conceptualizar la alianza entre paciente y terapeuta, partiendo por la definición de Freud (1912/1958), en la cual se entendía como una transferencia positiva necesaria para la efectividad de las interpretaciones del analista, pasando por la transferencia racional (Fenichel, 1936/1941), la alianza terapéutica (Zetzel, 1956) y la alianza de trabajo (Greenson, 1965). Sin embargo, Strupp (1973) fue el primero en concebirla como un constructo transversal a las distintas intervenciones. Siguiendo esta misma línea, Bordin (1979) agrega que es un constructo multidimensional que implica un acuerdo en las tareas y los objetivos del tratamiento y la necesidad de un vínculo positivo entre paciente y terapeuta (Bambling, King, Raue, Schweitzer \& Lambert, 2006; Cecero, Fenton, Frankforter, Nich \& Carrol, 2001). El paciente debe ser capaz de reconocer desde el inicio que puede contar con el apoyo del terapeuta, sobre todo en el caso de los adolescentes, quienes durante las primeras sesiones suelen necesitar desesperadamente una relación cálida, confiable y segura, que los haga sentir aceptados y entendidos (Eyrich-Garg, 2008; Martin, 2003; Sylwester, 2007). La sensación de un trabajo conjunto suele desarrollarse en las fases posteriores (Luborsky, 1976).

Lo cierto es que el trabajo terapéutico con adolescentes requiere de creatividad y flexibilidad en el uso de las técnicas, tomando en cuenta los elementos propios de esta etapa del desarrollo. Resulta importante, por ejemplo, conocer el desarrollo neurofisiológico de los adolescentes (Badenoch, 2008) y su desarrollo psicosocial, con el fin de comprender cómo influyen estas variables en el quehacer terapéutico (Faw, Hogue, Johnson, Diamond \& Liddle, 2005; Shirk \& Karver, 2011). De lo contrario, se estaría dando por hecho que la única forma de intercambiar información, contactarse con las emociones negativas y solucionar los problemas, es a través del intercambio verbal. De hecho, los adolescentes suelen llegar a terapia involuntariamente (Baillargeon, Coté \& Douville, 2012; Baylis, Collins \& Coleman, 2011; Bolton Oetzel \& Scherer, 2003; Horvath, 2009), con poca consciencia sobre sus problemas, baja motivación para involucrarse (Wright, Everett \& Roisman, 1986) y con la idea de no ser escuchados por los adultos. Suelen percibir al terapeuta como alguien que intentará controlarlos, darles consejos correctivos e instruirlos sobre cómo comportarse (Martin, 2003). Por ello, entregarles cierto grado de control y responsabilidad a lo largo del proceso termina convirtiéndose en uno de los componentes más favorecedores de la alianza terapéutica (Constantino, Castonguay, Zack \& DeGeorge, 2010; Fitzpatrick \& Irannejad, 2008; Karver et al., 2006). Por lo tanto, entre los desafíos más comunes están: acercarse al problema y trabajarlo conjuntamente, motivar al adolescente para mantener su compromiso, establecer un marco común para elaborar significados y manejar su ambivalencia (Binder, Holgersen \& Nielsen, 2008). Otro elemento diferenciador y enriquecedor que permite conceptualizar la alianza terapéutica con adolescentes es el vínculo que se establece con sus padres 
o cuidadores (Hogue, Daubter, Stambaugh, Cecero \& Liddle, 2006; McLeod \& Weisz, 2005; Shelef, Diamond, Diamond \& Liddle, 2005).

La manera de estudiar la alianza terapéutica también se ha visto impactada por el cambio de paradigma que subraya la importancia de los aspectos relacionales durante el tratamiento (Bambling et al., 2006; Horvath, 2009; Safran \& Muran, 2000/2005; Tryon, Blackwell \& Hammell, 2007). Así, existen investigaciones focalizadas en los factores que permiten comprender los momentos de ruptura y resolución de la alianza (Safran, Muran, Samstag \& Stevens, 2002) y su impacto en los resultados terapéuticos (Safran, Muran \& Eubanks-Carter, 2011), teniendo claro que la interacción entre paciente y terapeuta es un proceso relacional en el cual se influyen mutuamente (Sameroff, 2009; Staudt, 2007).

Los momentos de ruptura están asociados con activaciones disfuncionales de los patrones interpersonales del paciente que, cuando no son debidamente resueltos, terminan deteriorando la alianza y llevando muchas veces a resultados poco exitosos y/o al abandono de la terapia (Safran \& Kraus, 2014). Existen dos tipos de rupturas: (a) de retirada, caracterizadas por la negación de un estado anímico, respuestas mínimas, cambios abruptos de tema, e intelectualización, entre otras y (b) de confrontación, cuando existen quejas directas hacia la persona del terapeuta, hacia sus competencias, hacia las actividades propias de la terapia, o bien, hacia los logros durante la terapia (Safran \& Muran, 2000/2005). Ante estas situaciones, el terapeuta debe poder identificar e intentar resolver las rupturas conjuntamente con su paciente, a través de estrategias de resolución tales como invitar a expresar sentimientos negativos, aclarar malentendidos o vincular la ruptura con patrones interpersonales dentro y fuera de la sesión (Safran \& Kraus, 2014).

El dinamismo de la alianza terapéutica ha despertado el interés de investigar procesos terapéuticos con adolescentes, en términos de rupturas y resoluciones. Por ejemplo, se ha observado que los adolescentes más ambivalentes frente al cambio suelen ser menos receptivos a los intentos del terapeuta por resolver las rupturas (Hunter, Button \& Westra, 2014), las cuales suelen estar relacionadas con el nivel de compromiso del adolescente con su proceso, su conexión emocional, su sensación de seguridad y con el sentimiento de un propósito compartido con su familia (Escudero, Boogmans, Loots \& Friedlander, 2012). En este sentido, estos momentos de resolución conjunta se convierten en un ingrediente importante para el éxito del tratamiento (Daly, Llewelyn, Mdougall \& Chanen, 2010), sobre todo, si experimentan en la relación terapéutica conexión emocional y cognitiva expresada a nivel relacional, que favorece el desarrollo de un vínculo confiable para trabajar positiva y colaborativamente (Fitzpatrick \& Irannejad, 2008; Karver et al., 2006; Martin, 2003). Por lo tanto, es deber del terapeuta explorar el origen de las rupturas desde las expectativas, creencias y emociones del adolescente, ya que generalmente son un reflejo de cómo resuelven las tensiones interpersonales en su vida cotidiana (Baillargeon et al., 2012).

Si bien la mayoría de los estudios refleja distintas conceptualizaciones teóricas y metodológicas, los resultados indican que los momentos de ruptura y resolución son mecanismos esenciales que contribuyen al desarrollo y mantenimiento de la alianza a lo largo de la terapia, independientemente del modelo terapéutico.

Sobre esta base, el presente estudio se propuso lo siguiente: (a) caracterizar los momentos de ruptura identificados a lo largo del proceso terapéutico de un caso clínico, (b) analizar las estrategias utilizadas por la terapeuta con la intención de resolver dichas rupturas conjuntamente durante la sesión y (c) explicar clínicamente el impacto de ambos momentos en los resultados terapéuticos.

\section{Método}

\section{Diseño}

El presente estudio de caso único se basó en un diseño descriptivo-longitudinal, utilizando simultáneamente técnicas cualitativas y cuantitativas para la producción/recolección de datos. Por un lado, las sesiones fueron observadas por jueces entrenados y por terapeutas. Los resultados obtenidos se analizaron y discutieron con la técnica consensual qualitative research (CQR; Hill et al. 2005), para asegurar que fueran producto del acuerdo intersubjetivo. Por otro lado, se utilizaron cuestionarios de autorreporte con la validez estadística y la sensibilidad clínica necesarias para monitorear los resultados terapéuticos en distintos momentos del proceso. A partir de este diseño se esperaba reducir la brecha entre clínicos e investigadores, además de aportar elementos que enriquezcan el marco clínico y empírico que sustenta el trabajo terapéutico con adolescentes (Lingiardi, Gazzilo \& Wladron Jr., 2010). 


\section{Descripción del Caso}

Daniela tenía 13 años cuando fue derivada a psicoterapia por su psiquiatra, con quien había estado en tratamiento farmacológico durante el último año. Su caso era parte de la muestra recolectada por el autor principal de este artículo, como parte del Proyecto de Postdoctorado FONDECYT 3130367, titulado Características de la Comunicación Verbal de Pacientes con Depresión (Introyectivos y Anaclíticos) Durante la Conversación Terapéutica y su Relación con el Cambio Psicoterapéutico, cuyo objetivo era analizar procesos terapéuticos completos de adolescentes diagnosticados con difusión de identidad.

Daniela se encontraba desescolarizada al inicio del tratamiento (terminando $8^{\circ}$ básico), con clases particulares en casa y con exámenes libres. Vivía con su padre, madre y hermana, y no había antecedentes familiares de trastornos mentales. Dos años antes, Daniela y su familia se trasladaron de su ciudad de origen a la capital, lo que le generó muchas dificultades para adaptarse. Se eligió este caso por ser representativo del trabajo terapéutico realizado con adolescentes diagnosticados con difusión de identidad.

Historia y evolución del problema. Daniela presentaba problemas en sus relaciones sociales, específicamente, desde la enseñanza básica, cuando solía mostrarse dominante con sus compañeros de colegio. Asimismo, presentaba un patrón relacional que la llevaba a idealizar a sus amistades, mostrándose dependiente de las mismas; sin embargo, cuando se frustraba (e.g., que una amiga no hiciera lo que ella quería), terminaba devaluando y rompiendo el vínculo. Según sus padres, desde chica mostró una alta sensibilidad a la crítica y poca tolerancia a la frustración, que aumentó con el cambio de ciudad y el ingreso al nuevo colegio, donde fue víctima de bullying. Sus padres deciden cambiarla de colegio nuevamente, pero sigue mostrándose desconfiada y temerosa de volver a experimentar bullying, respondiendo agresivamente a cualquier señal que fuera interpretada por ella en esta línea. Finalmente, abandonó el colegio, eliminó todo contacto con sus amigos (Facebook, WhatsApp) y se encerró en su casa. Todo intento de acercamiento activaba nuevamente su desconfianza ("lo hacen por obligación, pero me odian en secreto"). Empezó a mostrarse más irritable (gritos a ambos padres y golpes a la madre) e irrespetuosa frente a la autoridad, lo que terminó desbordando a sus padres (su padre utilizó golpes para intentar calmarla). Carecía de una visión coherente y continua de sí misma y empezó a volverse más dependiente de su madre. Presentaba, además, conductas autolesivas (cortes en ambos brazos), ideación suicida, insomnio, inapetencia, anhedonia y llanto la mayor parte del tiempo. De acuerdo a la Entrevista Neuropsiquiátrica Internacional (MINI-Kid) realizada por el psiquiatra, Daniela presentaba un episodio depresivo mayor (F32.x) con riesgo suicida leve (F34.1) y fobia social (F40.1). También presentaba indicadores suficientes para un trastorno de personalidad borderline de acuerdo a la Entrevista Estructurada para el Eje II del DSM-IV (SCID-II), sin presencia de un trastorno antisocial de la personalidad.

Intervención psicoterapéutica. Daniela recibió un tratamiento de nueve meses que incluyó sesiones individuales (bisemanal), familiares (quincenal) y control farmacológico (quincenal). Las intervenciones se basaron en el modelo de tratamiento para la identidad del adolescentes (AIT; Foelsch et al., 2014), que adapta la psicoterapia focalizada en la transferencia (TFP) para el tratamiento de adolescentes. Este modelo fue desarrollado para tratar a quienes tienen o están en riesgo de desarrollar una patología grave de la personalidad. Se centra en mejorar la integración de identidad, aumentar el funcionamiento adaptativo, aumentar la productividad hacia el logro de objetivos de vida y el comportamiento en las áreas de la autorregulación y las relaciones interpersonales. Se realizó un contrato terapéutico, psicoeducación, pautas de crianza con los padres e intervenciones ambientales, con el objetivo de contener las conductas autolesivas graves y centrar la psicoterapia en la experiencia del sí mismo y la mejora de la regulación afectiva y conductual.

El proceso psicoterapéutico. Daniela y su terapeuta individual (psicóloga) lograron vincularse rápidamente y establecer objetivos terapéuticos de forma conjunta. Su poca tolerancia a la frustración quedó en evidencia desde las primeras sesiones, así como la falta de diferenciación de sí misma y los otros. Fue evidente su oscilación entre idealización-devaluación, tanto en las relaciones extra-transferenciales (e.g., amigas, padres) como con su terapeuta, lo que se evidenció en muchos momentos en los cuales se vio tensionada la alianza terapéutica. Al segundo mes de tratamiento y con una evolución sintomática favorable, Daniela realizó un viaje de intercambio cultural de dos meses, lo que fue considerado un hito importante dentro del tratamiento, ya que pudo establecer relaciones sociales y lograr un sentido de autoeficacia para enfrentar diferentes situaciones. Al volver, se reintegró al colegio de una manera mucho más adaptativa, pero sus relaciones sociales seguían basadas en la desconfianza y la proyección de sus conflictos internos. Este fue 
el eje central de las sesiones individuales, en las cuales se clarificaron, confrontaron e interpretaron contenidos, tanto extra-transferencialmente como en la relación terapéutica misma. Al quinto mes de tratamiento, mostró una mejoría progresiva (sintomatología, relaciones interpersonales y rendimiento académico), por lo que se disminuyeron progresivamente algunos elementos terapéuticos (una sesión por semana). Al final del tratamiento, se mantuvo la mejoría lograda, evidenciándose una visión más integrada de sí misma y de los otros, así como una mejor regulación de sus afectos y conductas. El tratamiento farmacológico finalizó un mes después de la finalización de la terapia.

\section{Recolección y Análisis de Datos}

Estrategia cuantitativa. Se utilizaron cuatro instrumentos, aplicados a Daniela por un ayudante de investigación (psicólogo).

Youth Outcome Questionnaire-Self Report (Y-OQ-SR). Este cuestionario fue desarrollado por Wells, Burlingame y Rose (2003) y se encuentra en proceso de validación con población chilena (Valdés \& Hernández, 2017). Consta de 64 ítems que evalúan el nivel de estrés psicosocial de pacientes entre 12 y 18 años, a partir de las siguientes áreas: malestar intrapersonal (ansiedad, depresión, desesperanza; 18 ítems; e.g., "Estoy tenso y me sobresalto fácilmente"); quejas somáticas (mareo, dolor de cabeza y estómago; 8 ítems; e.g., "Tengo dolores de cabeza o mareos"); relaciones interpersonales (discutir, desafiar, problemas de comunicación; 10 ítems; e.g., "Discuto o soy irrespetuoso con los demás"); problemas sociales (delincuencia o conducta agresiva; 8 ítems; e.g., "Amenazo con irme de la casa o lo he hecho"); disfunción del comportamiento (organización, concentración, manejo de la frustración y síntomas relacionados con el déficit atencional; 11 ítems; e.g., "Me resulta difícil esperar mi turno en actividades o conversaciones") e ítems críticos (ideas paranoicas, alucinaciones, manía e ideas suicidas; 9 ítems; e.g., "Creo que algunas personas tratan de lastimarme aun cuando sé que esto no es verdad"). Completar el instrumento toma 7 minutos y se responde en una escala Likert de 5 puntos (nunca a siempre), de modo que un puntaje total alto indica un funcionamiento disfuncional. El instrumento original tiene un punto de corte de 47 para el puntaje total. Ha mostrado una alta consistencia interna para el puntaje total (alfa de Cronbach $=0,96$ ) y entre 0,73 y 0,91 para las subescalas (Burlingame et al., 2005).

Cuestionario de Desarrollo de la Identidad en Adolescentes (AIDA). Este cuestionario de autorreporte fue desarrollado por Goth et al. (2012) y está conformado por 58 ítems que evalúan la capacidad de adolescentes entre 12 y 20 años para definirse a sí mismos de manera continua (atributos, relaciones interpersonales y autorreflexión emocional; e.g., "He tenido los mismos hobbies e intereses, los que me hacen ser como soy") y coherente (consistencia en el autoconcepto, autonomía y autorreflexión cognitiva; e.g., "Siento que tengo caras diferentes, que juntas no encajan bien"). Los ítems se responden en una escala Likert de 5 puntos (muy en desacuerdo hasta muy de acuerdo). Un puntaje por encima del punto de corte (60) indica una identidad poco integrada o difusa. El AIDA fue adaptado y validado en Chile por Valdés, Hernández, Goth, Quevedo y Borzutzky (2018), mostrando una alta consistencia interna para la escala total (alfa de Cronbach $=0,94)$ y para las subescalas de Continuidad y Coherencia $(0,88$ y 0,91 , respectivamente), así como una alta correlación directa $(0,72, p<0,001)$ con la Subescala A del Inventario Clínico para Adolescentes de Millon (Vinet \& Santacana, 2008).

Inventario de Depresión de Beck - Forma I (BDI-I). Fue desarrollado por Beck, Ward, Mendelson, Mock y Erbaugh (1961). Evalúa la sintomatología depresiva y consta de 21 categorías de síntomas y actitudes (14 evalúan síntomas cognitivos y afectivos; e.g., "Me siento culpable buena parte del tiempo" y 7 evalúan síntomas somáticos y conductuales; e.g., "Puedo dormir tan bien como antes"), con cuatro o cinco afirmaciones en orden creciente de severidad. El BDI-I fue validado en Chile por Valdés et al. (2017), mostrando una alta consistencia (alfa de Cronbach =0,92), alcanzando valores de 0,91 y 0,93 en la muestra del análisis factorial exploratorio y confirmatorio, respectivamente. Según el método de Youden, el puntaje de corte para población chilena que indica presencia de depresión es 11,5, con una especificidad de 0,86 y una sensibilidad de 0,91.

Inventario de Resolución de Problemas Revisado (SPSI-RS, forma breve, D'Zurilla, Nezu \& Maydeu-Olivarez, 1999). Evalúa la capacidad del paciente para resolver problemas, a partir de 25 ítems agrupados equitativamente (cinco ítems) en cinco factores para cada dimensión: funcional (orientación positiva al problema, OPP; resolución racional al problema, RRP; e.g., "Cuando resuelvo problemas, se me ocurren muchas opciones") y disfuncional (orientación negativa al problema, ONP; estilo 
impulsivo/descuidado, EID; estilo evitativo, EE; e.g., "Dudo que sea capaz de resolver problemas difíciles no importa lo mucho que lo intente"). Los ítems se responden en una escala Likert de 5 puntos (no es cierto en absoluto hasta extremadamente cierto). Se utilizó la versión española de Calero, Luna, Vera-Villarroel y González (2001), la cual ha reportado propiedades psicométricas satisfactorias, con una consistencia interna de magnitud adecuada para las distintas subescalas (alfa de Cronbach entre 0,64 y 0,82) y una estructura factorial similar a la de la versión original.

Las diferencias en las puntuaciones obtenidas (pre y post-test) fueron estimadas a partir del error estándar de medición (EEM). Para determinar la existencia de diferencias estadísticas entre dos proporciones, se utilizó el puntaje $Z$-ratio para proporciones independientes.

Estrategia cualitativa. Un total de 32 sesiones grabadas en audio y video fueron observadas por dos jueces (un psicólogo clínico y un estudiante de doctorado en psicoterapia) entrenados en el Sistema de Identificación de Rupturas y Resoluciones (3RS; Eubanks et al., 2015), el cual permite la identificación de momentos caracterizados por una tensión en la relación colaborativa entre paciente y terapeuta. Los momentos de ruptura varían en su intensidad: desde una vaga consciencia de la falta de colaboración hasta deterioros mayores en la alianza terapéutica. Pueden ser de dos tipos: por distanciamiento (cuando el paciente se desconecta parcialmente de su terapeuta, de sus propias emociones o de algunos aspectos del proceso terapéutico) y por confrontación (cuando el paciente se mueve contra su terapeuta, ya sea expresando ira o insatisfacción). En ambos casos, se altera la colaboración, lo que hace necesario utilizar estrategias para intentar resolver la ruptura; sin embargo, no siempre se logra.

El entrenamiento de los jueces incluyó la revisión del manual, codificaciones individuales cada semana, y la discusión posterior con el equipo de investigación. La capacitación finalizó cuando ambos jueces alcanzaron un alto grado de acuerdo en sus codificaciones. Se calcularon los índices Kappa de Cohen, considerando la totalidad de ventanas con presencia de un evento relevante $(N=61)$. Se observó un grado de acuerdo casi perfecto entre los jueces y la terapeuta del caso, así como con otro terapeuta externo (ver Tabla 1).

Tabla 1

Resultados del Estudio de Confiabilidad entre Jueces

\begin{tabular}{|c|c|c|c|c|c|c|c|c|c|}
\hline & \multicolumn{3}{|c|}{$\begin{array}{l}\text { Observadores } \\
\text { entrenados }\end{array}$} & \multicolumn{3}{|c|}{$\begin{array}{c}\text { Observadores entrenados } \\
\text { y terapeuta A * }\end{array}$} & \multicolumn{3}{|c|}{$\begin{array}{c}\text { Observadores entrenados } \\
\text { y terapeuta } \mathrm{B} * *\end{array}$} \\
\hline & Kappa & $p$ & Nivel & Kappa & $p$ & Nivel & Kappa & $p$ & Nivel \\
\hline $\begin{array}{l}\text { Momentos de } \\
\text { ruptura }\end{array}$ & 1,000 & $<0,001$ & Perfecto & 0,940 & $<0,001$ & $\begin{array}{c}\text { Casi } \\
\text { perfecto }\end{array}$ & 0,911 & $<0,001$ & $\begin{array}{c}\text { Casi } \\
\text { perfecto }\end{array}$ \\
\hline \multicolumn{10}{|l|}{ Tipos de ruptura } \\
\hline Distanciamiento & 0,740 & $<0,001$ & Substancial & & & & & & \\
\hline Confrontación & 0,831 & $<0,001$ & $\begin{array}{c}\text { Casi } \\
\text { perfecto }\end{array}$ & & & & & & \\
\hline $\begin{array}{l}\text { Contenido de las } \\
\text { rupturas }\end{array}$ & 0,788 & $<0,001$ & Substancial & & & & & & \\
\hline $\begin{array}{l}\text { Intentos de } \\
\text { resolución }\end{array}$ & 0,967 & $<0,001$ & $\begin{array}{c}\text { Casi } \\
\text { perfecto }\end{array}$ & 0,914 & $<0,001$ & $\begin{array}{c}\text { Casi } \\
\text { perfecto }\end{array}$ & 0,913 & $<0,001$ & $\begin{array}{c}\text { Casi } \\
\text { perfecto }\end{array}$ \\
\hline $\begin{array}{l}\text { Estrategias de } \\
\text { resolución }\end{array}$ & 0,934 & $<0,001$ & $\begin{array}{c}\text { Casi } \\
\text { perfecto }\end{array}$ & & & & & & \\
\hline
\end{tabular}

Nota. * Terapeuta de Daniela; ** Terapeuta externo entrenado. Los resultados fueron interpretados de la siguiente manera: valores de Kappa $\leq 0$ indican ausencia de acuerdo, 0,01 a 0,20, escaso, 0,21 a 0,40, suficiente, 0,41 a 0,60, moderado, 0,61 a 0,80, substancial, 0,81 a 0,99, casi perfecto y 1,00 , perfecto.

\section{Procedimiento}

Se invitó a los padres de Daniela a participar en el estudio, quienes, en conjunto con la terapeuta del caso, firmaron el consentimiento y el asentimiento informado, una vez aceptados todos los compromisos y condiciones. Este estudio empezó a implementarse una vez que fue aprobado por el Comité Ético de Ciencias Sociales, Artes y Humanidades de la Pontifica Universidad Católica de Chile y por el Comité Asesor de Bioética de FONDECYT. Antes de iniciar el tratamiento, se llevó a cabo una evaluación inicial que contempló 
la realización de una entrevista neuropsiquiátrica (MINI-Kid) y una entrevista estructurada (SCID-II) por parte de un psiquiatra. Además, se aplicaron en dos momentos (pre y post tratamiento) los siguientes instrumentos: Y-OQ-SR, AIDA, BDI-I y SPSI-RS. Todas las sesiones fueron grabadas en audio y video, las que fueron transcritas para su posterior análisis.

\section{Resultados}

\section{Resultado Terapéutico}

Sintomatología. Al inicio del tratamiento Daniela se ubicaba dentro de la población disfuncional $(\mathrm{PC}=47)$, según los resultados obtenidos en el Y-OQ-SR (ver Figura 1), con puntajes por encima de los puntos de corte en todas las subescalas. Reportaba una alta disconformidad con su calidad de vida, pobres relaciones sociales y alta sintomatología, lo que daba cuenta de la complejidad del caso.

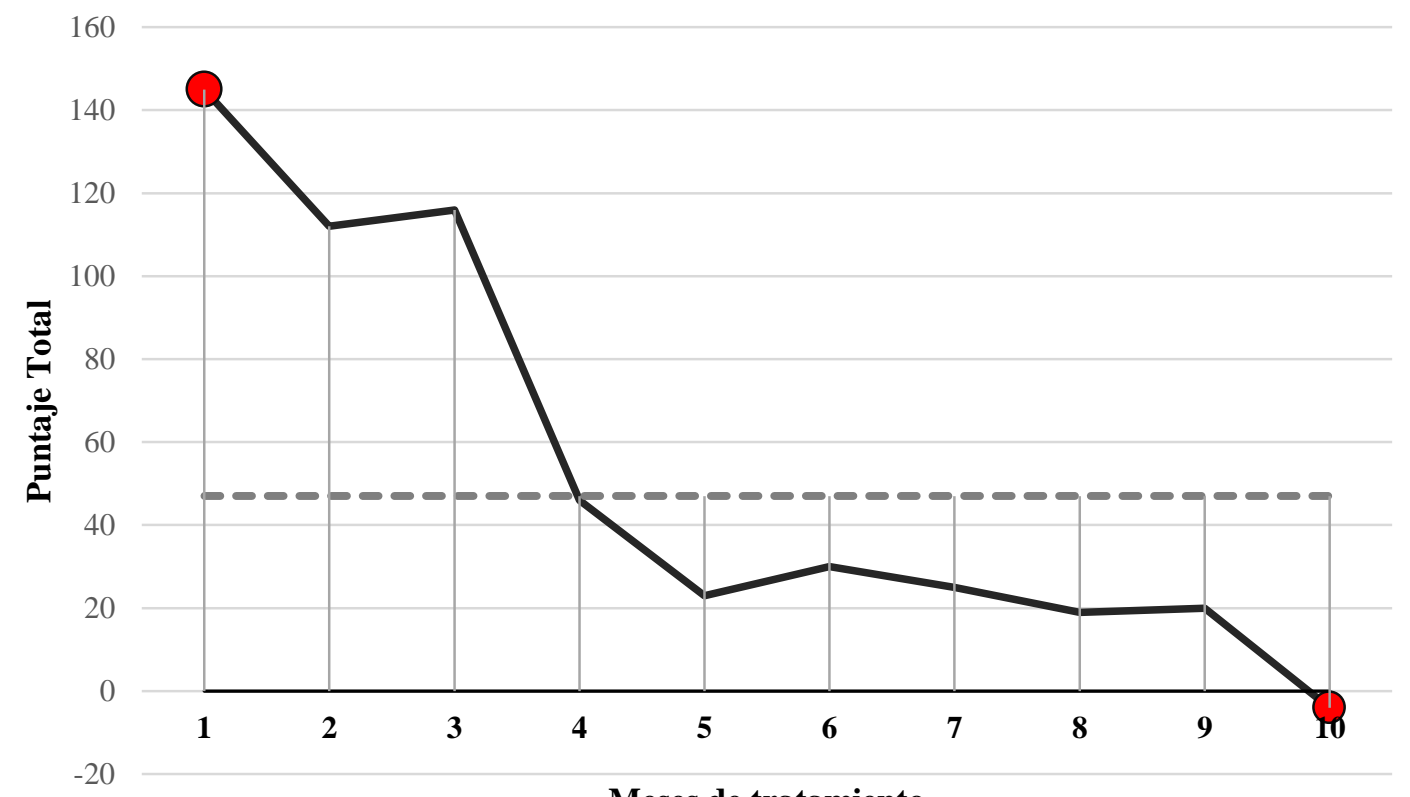

Meses de tratamiento

Figura 1. Resultado psicoterapéutico a partir del puntaje total del Y-OQ-SR 2.0. La línea puntuada corresponde al punto de corte.

Al finalizar la terapia, mostró una evidente mejoría y un consecuente cambio subjetivo frente a sus síntomas, el cual empezó a evidenciarse al $2^{\circ}$ mes de tratamiento. Sin embargo, estos fueron clínica y estadísticamente más significativos al $4^{\circ}$ mes de tratamiento, cuando disminuyó completamente la sintomatología que la aquejaba (EEM $= \pm 12,1)$. Estos resultados se condicen con los observados en el BDI-I, que reportaron una sintomatología depresiva grave al inicio (32 puntos), que no solo se redujo durante el tratamiento, sino que desapareció completamente.

Resolución de problemas. Sus capacidades menos adaptativas para la resolución de problemas se mostraron más activas al inicio del tratamiento $(\mathrm{ONP}=16$, EID $=13)$, con respecto a aquellas más adaptativas $(\mathrm{OPP}=4 \mathrm{y} R \mathrm{RP}=2)$ (ver Figura 2). Esta situación se invierte al finalizar el tratamiento, $\mathrm{y}$ Daniela muestra una mayor orientación positiva al problema $(E E M= \pm 3,83)$ y una mayor respuesta racional hacia los mismos $(\mathrm{EEM}= \pm 3,61)$. Esto coincide con el hecho de encontrarse menos sintomática y con un menor malestar psicológico subjetivo. A pesar de que no hubo una diferencia estadística en relación al estilo evitativo $(\mathrm{EEM}= \pm 3,6)$, la paciente dejó de retrasar la toma de decisiones y de afrontar los problemas. 


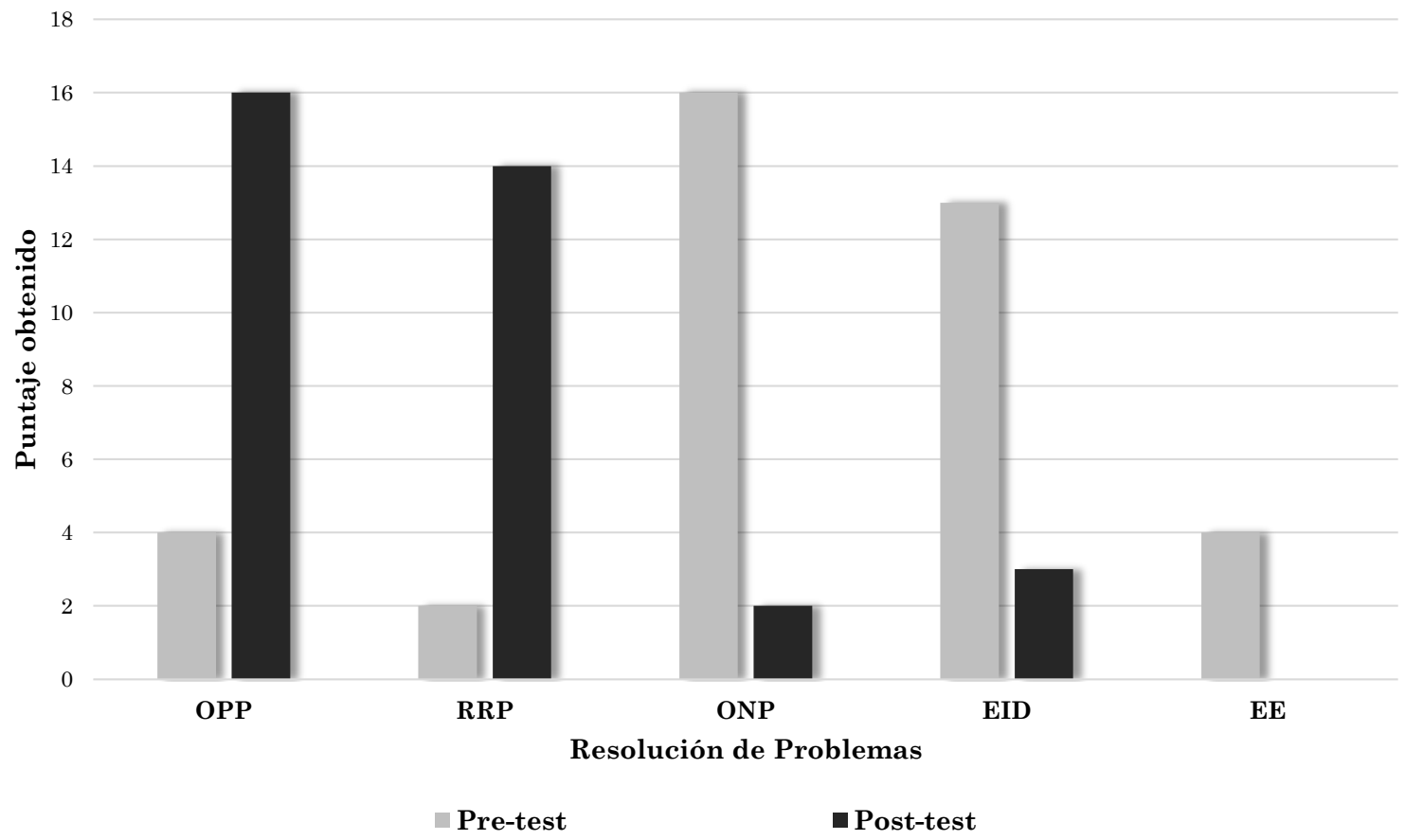

Figura 2. Resultados en la resolución de problemas según el SPSI-R: OPP = Orientación positiva del problema, ONP = Orientación negativa del problema, EID = Estilo impulsivo y descuidado, EE = Estilo de evitación y RRP = Resolución racional del problema.

Desarrollo de la identidad. Daniela obtuvo un puntaje total de 76 puntos en el AIDA antes de iniciar el tratamiento, estando a dos desviaciones estándar por encima del promedio $(\mathrm{PC}=60)$. Tal como lo muestra la Figura 3, este puntaje disminuyó significativamente al final del tratamiento $(\mathrm{EEM}= \pm 17,8)$.

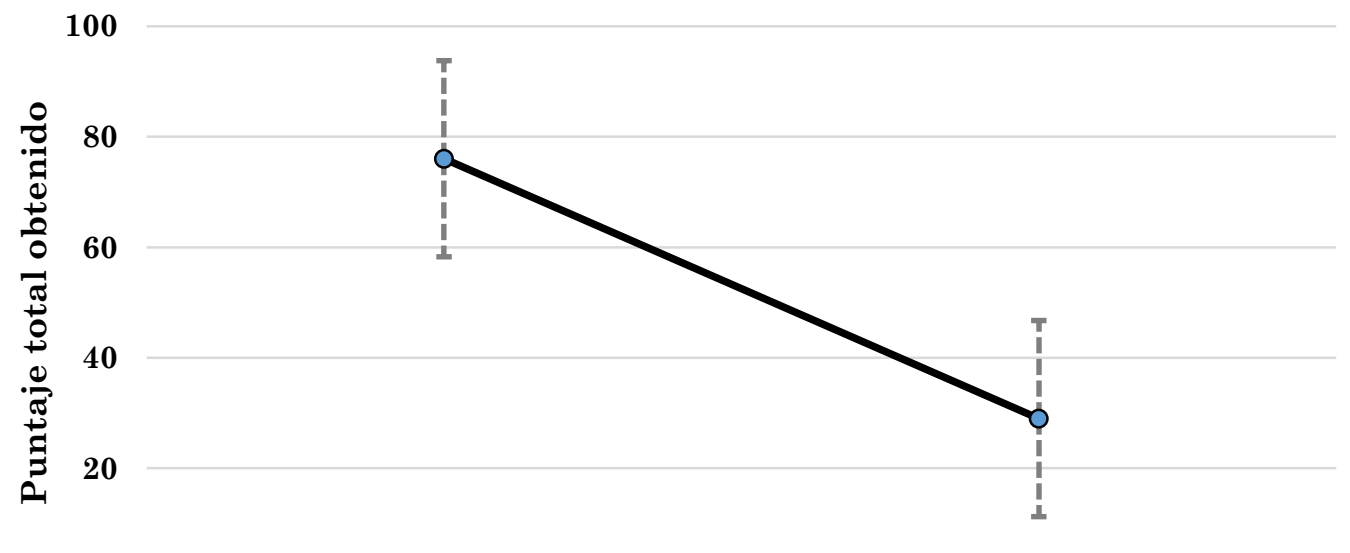

0

Baseline

Postline

\section{Desarrollo de Identidad}

Figura 3. Puntajes obtenidos al inicio y al final del tratamiento en el desarrollo de la identidad según el AIDA. 


\section{Rupturas e Intentos de Resolución de la Alianza Terapéutica}

Para la presente investigación se agruparon en clusters los distintos tipos de ruptura y estrategias de resolución, tal como se muestra en la Tabla 2. Se analizaron únicamente los momentos en los cuales hubo total acuerdo entre ambos jueces y con los terapeutas. Todas las sesiones $(N=32)$ fueron subdivididas en ventanas de cinco minutos $(N=337$ ventanas). De estas, hubo 34 ventanas con la presencia de momentos de ruptura e intentos de resolución (11 rupturas de distanciamiento y 18 rupturas de confrontación).

Tabla 2

Rupturas e Intentos de Resolución Agrupados en Clusters

\begin{tabular}{|c|c|}
\hline Rupturas & Intentos de resolución \\
\hline \multicolumn{2}{|l|}{ Rupturas de distanciamiento } \\
\hline $\begin{array}{l}\text { 1. Negación, respuesta mínima y disociación (cluster } \\
\text { 1) } \\
\text { 2. Evitación y desconexión (cluster 2) } \\
\text { 3. Autocrítica y desesperanza (cluster 3) } \\
\text { 4. Paciente con una actitud deferente (cluster 4) }\end{array}$ & $\begin{array}{l}\text { 1. Invitar a expresar sentimientos negativos, aclarar } \\
\text { malentendidos y desconocimiento de alguna } \\
\text { información del paciente (cluster } 1 \text { ) } \\
\text { 2. Justificar y reestructurar las tareas de la terapia } \\
\text { (cluster 2) }\end{array}$ \\
\hline $\begin{array}{l}\text { Rupturas de confrontación } \\
\text { 5. Quejas dirigidas a la persona del terapeuta } \\
\text { (cluster 5) } \\
\text { 6. Quejas dirigidas hacia las intervenciones y tareas } \\
\text { (cluster 6) } \\
\text { 7. Quejas dirigidas hacia los parámetros generales } \\
\text { de la terapia (cluster 7) }\end{array}$ & $\begin{array}{l}\text { 3. Autorevelar su propia experiencia interna (cluster } \\
\text { 3) } \\
\text { 4. Vincular la ruptura con patrones interpersonales } \\
\text { en general (cluster 4) } \\
\text { 5. Justificar las defensas del paciente (cluster 5) }\end{array}$ \\
\hline
\end{tabular}

Momentos de ruptura de la alianza terapéutica. Con el fin de ilustrar cada uno de los tipos de ruptura y los intentos de la terapeuta para reparar dicha tensión en el vínculo, se incluyeron al final algunas viñetas extraídas a lo largo del proceso terapéutico (ver Anexo). Los primeros cuatro clusters corresponden a las rupturas de distanciamiento y los últimos tres, a las de confrontación.

Negación, respuesta mínima y disociación (cluster 1). Son actitudes y acciones por parte del paciente que disminuyen la calidad colaborativa del vínculo terapéutico, en tanto hay respuestas mínimas o incluso se guarda silencio durante la sesión, negando estados emocionales de forma no colaborativa con el terapeuta, y disociando el contenido entre lo que se dice y lo que se expresa no-verbalmente. En la sesión 15, se suscitó un momento de distanciamiento por parte de Daniela, en el que negaba verbalmente que existieran razones para sentirse molesta; sin embargo, su conducta no-verbal mostraba todo lo contrario. Frente a esto, la terapeuta la invitó a expresar sus sentimientos negativos para intentar resolver dicha ruptura (cluster 1 de estrategias de resolución), lo cual será ejemplificado en el apartado correspondiente.

Evitación y desconexión (cluster 2). Hace referencia a aquellos momentos en los cuales la comunicación se vuelve abstracta, siendo incapaz de vincular la propia experiencia con lo que se está diciendo en ese momento; o bien, cambiar de tema abruptamente, distraerse frecuentemente o manifestar abiertamente no querer hablar de un tema. En la sesión 13, Daniela muy molesta le expresó a la terapeuta no querer hablar de cierto tema por no querer recordar (ver viñeta 1). En ese momento, fue evidente su interés de evadir ciertas temáticas que la hacían sentir incómoda, solicitando explícitamente cambiar el foco de la conversación durante ese momento de la sesión.

Autocrítica y desesperanza (cluster 3). Hay otros momentos de la terapia en la cual el proceso colaborativo con el paciente disminuye, debido a que este último entra en una especie de proceso autocrítico y/o desesperanzador, que deja por fuera cualquier intento de ayuda por parte del terapeuta. Este marcador de ruptura queda ilustrado con la viñeta 2. 
Paciente en actitud deferente (cluster 4). Se refiere a un exceso de obediencia por parte del paciente, lo que hace que se muestre excesivamente acatador y en una actitud de sometimiento al terapeuta. En este caso no fue posible identificar este tipo de marcador.

Quejas dirigidas a la persona del terapeuta (cluster 5). En ocasiones, un paciente puede tener sentimientos negativos (e.g., rabia, impaciencia, desconfianza, rechazo) hacia su terapeuta o sentir que este le ha fallado. También es posible que critique explícitamente el estilo interpersonal del terapeuta, intente controlar sus intervenciones durante la sesión e, incluso, llegue a expresar abiertamente dudas relacionadas con su competencia profesional. Este marcador de ruptura es ejemplificado en la viñeta 3, en la cual Daniela solicita de manera muy confrontativa un cambio de terapeuta.

Quejas dirigidas hacia las intervenciones y tareas de la terapia (cluster 6). Este marcador incluye todas aquellas acciones realizadas explícita o implícitamente por el paciente, con el fin de rechazar el punto de vista del terapeuta, o bien, cualquier intervención y/o actividad propuesta durante la sesión. Tiene que ver con una actitud de desacuerdo no colaborativa. Este tipo de ruptura se ilustra en la viñeta 4: Daniela se oponía a trabajar su dificultad para expresarles a sus padres lo que pensaba y sentía. Esta situación activó en la terapeuta la necesidad de intentar resolver dicha ruptura, justificando su intervención a partir de las necesidades mismas de la terapia (marcador del cluster 2 de estrategias de resolución), lo cual no fue posible, ya que la respuesta de Daniela fue: "Bueno, si no les quieres decir, no les digas".

Quejas dirigidas hacia los parámetros generales de la terapia (cluster 7). Este último marcador incluye las quejas del paciente en relación a los objetivos, el setting y/o el progreso general de la terapia. Por ejemplo, Daniela llegó enojada a la sesión, expresando su deseo de disminuir el número de sesiones por semana (ver viñeta 5).

Intentos de resolución de la alianza terapéutica. La presencia de rupturas durante la terapia conllevó naturalmente a que la terapeuta, en la mayoría de los casos, intentara resolverlas inmediatamente en pos del vínculo y la colaboración por parte de la paciente. Para ello, utilizó una serie de estrategias que, para su análisis, también fueron agrupadas en los siguientes clusters.

Invitar a expresar sentimientos negativos, aclarar malentendidos y desconocimiento de alguna información del paciente (cluster 1). Fue la estrategia mayormente utilizada por la terapeuta con la intención de explorar juntas los problemas suscitados en la relación, expresar sentimientos difíciles de forma racional y/o clarificar información imprecisa o malentendida. Ante el momento de ruptura suscitado en la sesión 15 (cluster 1 de rupturas), la terapeuta recurrió inmediatamente a utilizar esta estrategia, tal como se muestra en la viñeta 6 .

Justificar y reestructurar las tareas de la terapia (cluster 2). Los desacuerdos relacionados con las tareas propias de la terapia se intentan resolver justificando o reestructurando las mismas, de tal forma que le haga más sentido al paciente. Esta estrategia fue utilizada por la terapeuta en el momento de ruptura suscitado en la sesión 13 (cluster 6 de rupturas), durante el cual hubo una confrontación directa de Daniela hacia algunas intervenciones realizadas durante el tratamiento (ver viñeta 7 ).

Autorrevelar su propia experiencia interna (cluster 3). Esta estrategia fue utilizada muy pocas veces por la terapeuta y se refiere a la capacidad de reconocer alguna responsabilidad en la ruptura suscitada; o bien, explicitar durante la conversación aspectos relacionados con su experiencia interna, por ejemplo, cuando la terapeuta en la sesión 15 le expresó a Daniela cómo se sentía ella debido a su actitud poco colaborativa durante las últimas sesiones (ver viñeta 8). Sin embargo, en esta ocasión la ruptura no fue resuelta y la paciente se mantuvo con la misma actitud durante toda la sesión (¿Tú crees que estoy haciendo esto para que le digas a mi papá cómo me siento? Yo no te estoy exigiendo nada, si no les quieres decir no les digas").

Vincular la ruptura con patrones interpersonales en general (cluster 4). En otros momentos, un terapeuta puede recurrir a explorar posibles conexiones entre una ruptura suscitada durante la sesión y situaciones específicas relacionales en la vida del paciente. La ventaja de esta estrategia es que, además de permitir resolver la ruptura, se provee al paciente de experiencias relacionales nuevas que le permitan darse cuenta de cuál suele ser su oferta relacional con otros y examinar sus propias reacciones y las de los demás, 
en el aquí y ahora. La viñeta 9 permite ejemplificar cómo la terapeuta aprovechó este momento de ruptura para conectarlo con patrones relacionales de Daniela, dentro y fuera del contexto terapéutico.

Justificar las defensas del paciente (cluster 5). Esta última estrategia fue utilizada por la terapeuta solamente una vez, cuando Daniela expresó que no tenía ganas de hablar de un tema particular que no quería recordar, aduciendo que tenía sueño y se sentía fastidiada (cluster 2 de las rupturas). En este momento, en lugar de desafiar su comportamiento defensivo, la terapeuta optó por validar el modo en que esta actitud le resultaba comprensible y adaptativa (ver viñeta 10 ).

\section{Dinámicas en la Alianza Terapéutica}

A diferencia del estudio realizado por Bennett, Parry y Ryle (2006), en el cual las rupturas eran inferidas a partir de un test que evaluaba la alianza terapéutica desde la percepción de los pacientes, en el presente estudio se consideró la percepción de la terapeuta encargada del caso, como una manera de complementar el análisis de proceso realizado por los observadores - momento a momento- de cada una de las sesiones. De esta forma, se toma en consideración la sugerencia realizada por algunos autores (Kazdin, 2008; Pachankis \& Goldfried, 2007) acerca de la necesidad de utilizar técnicas cualitativas para estudiar procesos psicoterapéuticos a un micro-nivel.

Se observó una mayor proporción de ventanas con momentos de ruptura por confrontación; sin embargo, la diferencia no alcanzó a ser estadísticamente significativa, $Z=1,838, p=0,066$ (ver Figura 4). La estrategia más frecuentemente utilizada por la terapeuta para intentar resolver los momentos de ruptura fue invitar a la paciente a expresar sentimientos negativos y aclarar malentendidos (ver Figura 5), independientemente del tipo de ruptura, $Z=0,707, p=0,480$. Tampoco hubo diferencia en la proporción de intentos de resolución por parte de la terapeuta y la proporción de rupturas, lo que da cuenta de la respuesta inmediata por parte de la terapeuta de emplear estrategias que les permitiera a ambas intentar resolver los desacuerdos.

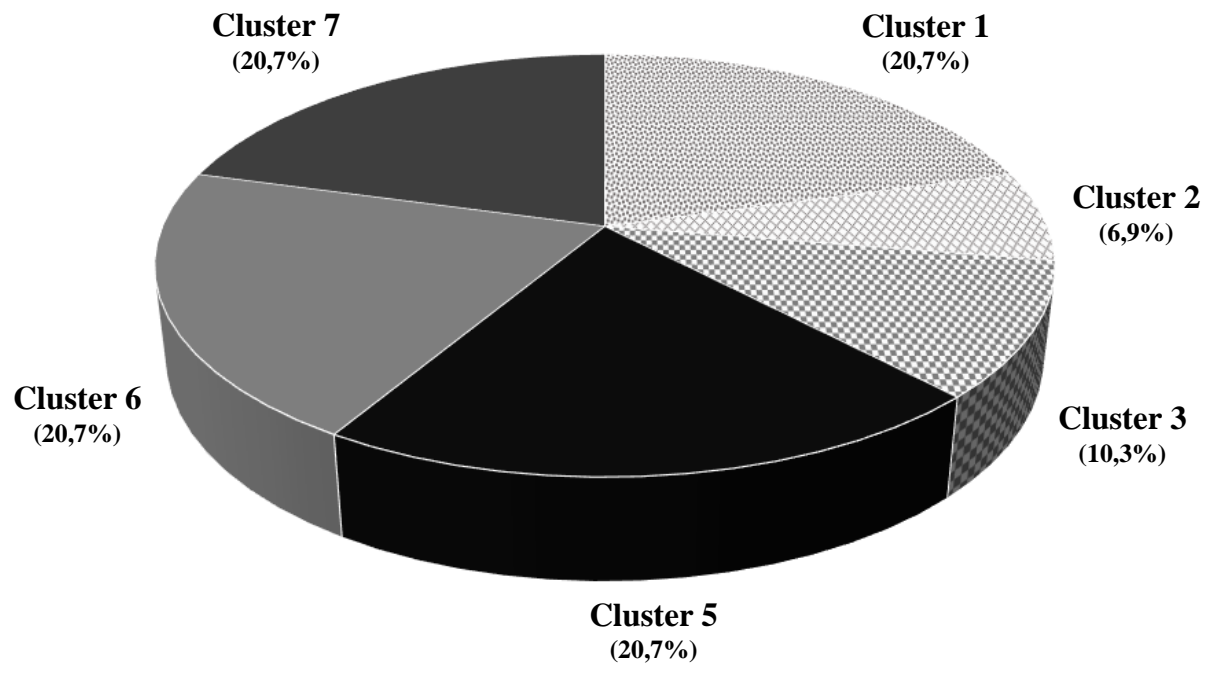

Figura 4. Distribución porcentual de los tipos de ruptura de la alianza terapéutica agrupados por clusters: Negación, respuesta mínima y disociación (cluster 1); Evitación y desconexión (cluster 2); Autocrítica y desesperanza (cluster 3); Actitud deferente (cluster 4); Quejas dirigidas a la persona del terapeuta (cluster 5); Quejas dirigidas hacia las intervenciones y tareas (cluster 6); Quejas dirigidas hacia los parámetros generales de la terapia (cluster 7). 


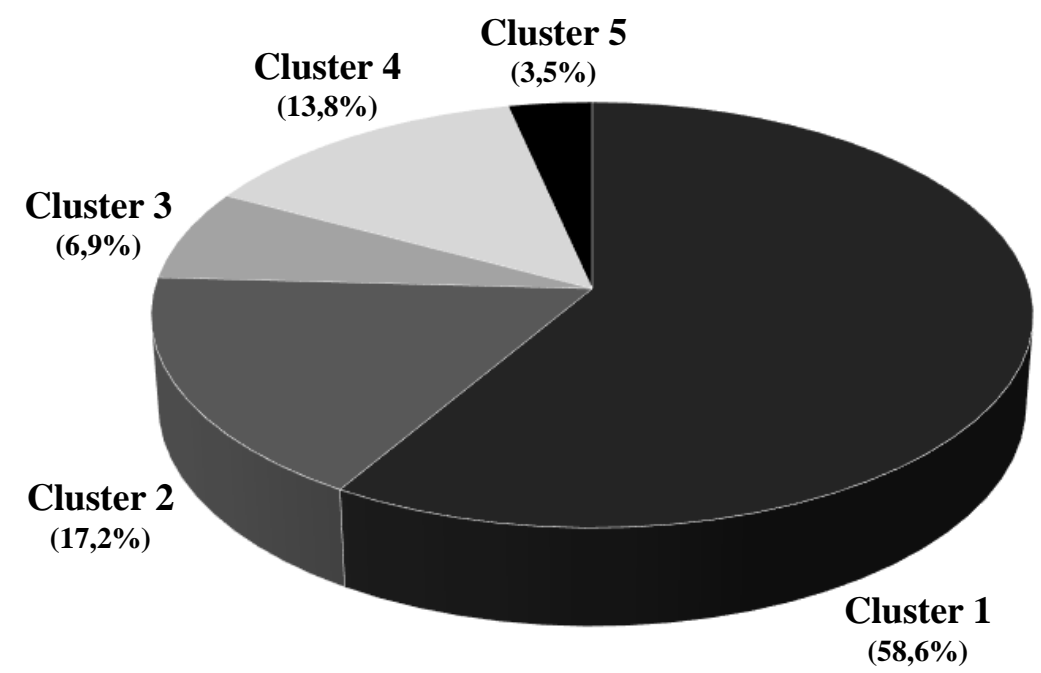

Figura 5. Distribución porcentual de los tipos de estrategias utilizadas por el terapeuta para intentar resolver la ruptura de la alianza terapéutica: Invitar a expresar sentimientos negativos, aclarar malentendidos y desconocimiento de alguna información del paciente (cluster 1); Justificar y reestructurar las tareas de la terapia (cluster 2); Autorevelar su propia experiencia interna (cluster 3); Vincular la ruptura con patrones interpersonales en general (cluster 4); Justificar las defensas del paciente (cluster 5).

La Figura 6 muestra la trayectoria de los episodios de ruptura e intentos de resolución por parte de la terapeuta — sesión a sesión - con tres fases claramente identificadas: la primera muestra una ausencia de momentos de tensión (sesiones 1 a 11); en la segunda hubo momentos de tensión en el vínculo, acompañados de intentos de resolución por parte de la terapeuta, sin una resolución explícita (sesiones 12 a 21); y en la tercera se observaron episodios de ruptura con una resolución explícita (sesiones 22 a 32).

Indistintamente del tipo de ruptura, estas surgieron cuando Daniela empezó a mostrar desagrado con la estructura terapéutica y con algunas intervenciones realizadas. La primera ruptura tuvo que ver con un evento vivenciado negativamente por Daniela durante la sesión de terapia familiar (realizada por otro terapeuta), previa a la sesión individual $\mathrm{N}^{\circ} 12$. Se puede observar cómo en la mayoría de los casos las rupturas de confrontación eran precedidas por rupturas de distanciamiento, lo que se interpretó como una imposibilidad de poner en palabras aquello que "nos está pasando", generándose una acumulación de experiencias negativas que terminaban tarde o temprano por conformar rupturas confrontacionales (Pos, Greenberg \& Warwar, 2009). En la segunda fase, si bien es cierto que los intentos de la terapeuta por resolver las rupturas no culminaron en resoluciones explícitas, es posible inferir que sí ayudaron a pavimentar el camino para posteriores resoluciones, coincidiendo esto con lo que Greenberg (1984) plantea como intentos parciales de solución, que dan cuenta de un proceso acumulativo y que, a la larga, terminaban igualmente teniendo un efecto en la autorregulación emocional por parte de la paciente.

Las resoluciones explícitas de las rupturas fueron más evidentes en la tercera fase, las cuales coinciden con la primera vez que la terapeuta explicita elementos contratransferenciales en la relación, permitiéndole a Daniela reconocer, comprender e integrar aquellas emociones negativas que había estado proyectando en su terapeuta durante las sesiones anteriores (Malan, 2007). Estos momentos específicos de la terapia fueron interpretados como instancias en las que la paciente tuvo la posibilidad de corregir experiencias emocionales, teniendo a la terapeuta como modelo y objeto a la vez (Newirth, 2000). 


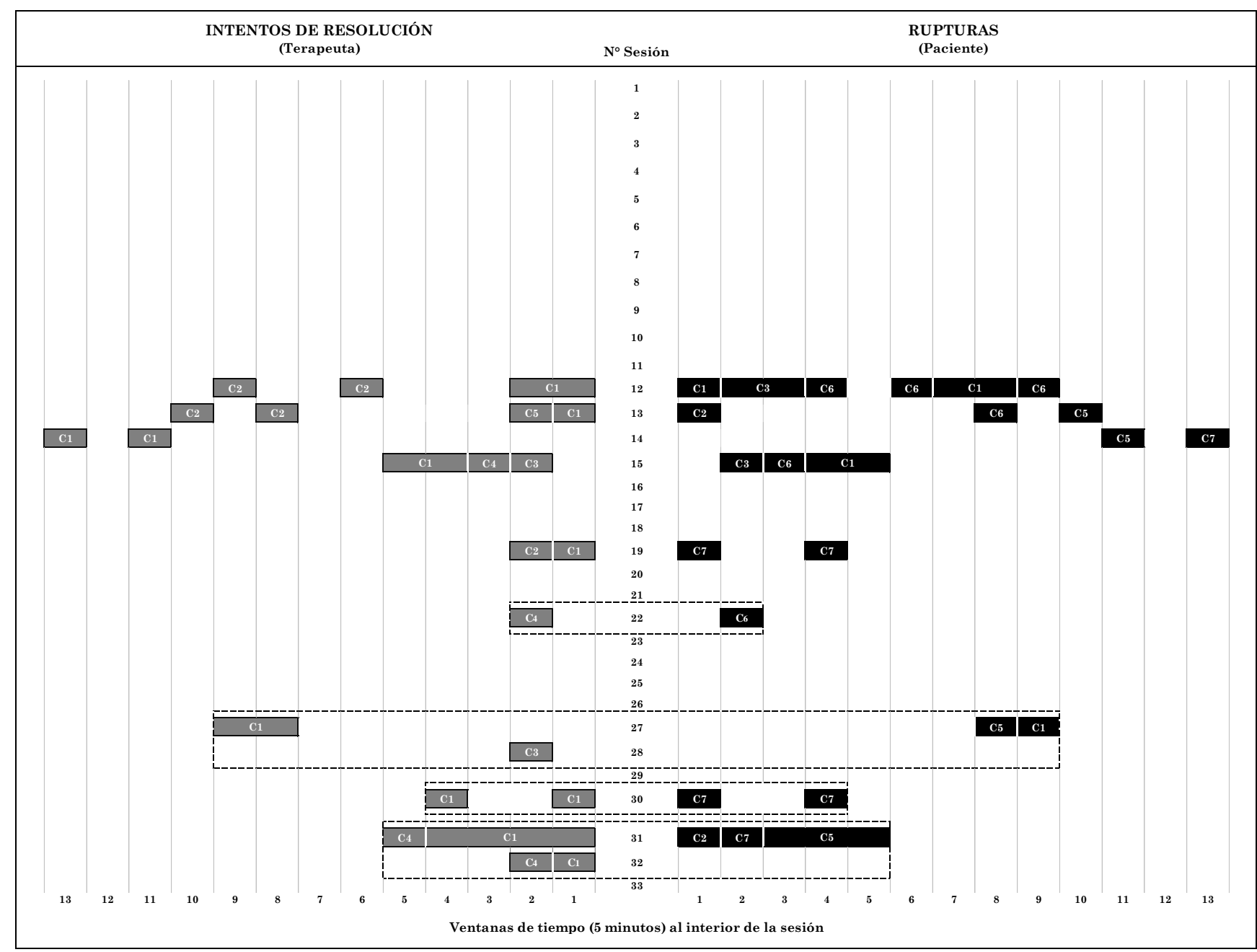

Figura 6. Distribución por sesión de los momentos de ruptura de la alianza terapéutica y las estrategias utilizadas por la terapeuta para intentar resolverlas. Clusters-Rupturas: negación, respuesta mínima y disociación $\left(\mathrm{C}_{1}\right)$; evitación y desconexión $\left(\mathrm{C}_{2}\right)$; autocrítica y desesperanza $\left(\mathrm{C}_{3}\right)$; quejas dirigidas a la persona del terapeuta $\left(\mathrm{C}_{5}\right)$; quejas dirigidas hacia las intervenciones y tareas $\left(\mathrm{C}_{6}\right)$; quejas dirigidas hacia los parámetros de la terapia $\left(\mathrm{C}_{7}\right)$. Clusters-Resolución: invitar a expresar sentimientos negativos y aclarar malentendidos $\left(\mathrm{C}_{1}\right)$; justificar y reestructurar las tareas de la terapia $\left(\mathrm{C}_{2}\right)$; autorevelar su propia experiencia interna $\left(\mathrm{C}_{3}\right)$; vincular la ruptura con patrones interpersonales $\left(\mathrm{C}_{4}\right)$; justificar las defensas del paciente $\left(\mathrm{C}_{5}\right)$.

\section{Discusión}

Las asociaciones entre el cambio de los pacientes y la relación terapéutica ha sido un tema ampliamente estudiado, existiendo un consenso acerca de la manera como determinados factores comunes terminan explicando el $8 \%$ de la varianza del resultado de la terapia, siendo importante la calidad, cualidad y la naturaleza de la alianza terapéutica (Horvath, 2006; Horvath, Del Re, Flückiger, \& Symonds, 2011; Platt, 2008; Safran \& Muran, 2000; Santibáñez Fernández, Román Mella \& Vinet, 2009), siendo importante la calidad, cualidad y la naturaleza de la alianza terapéutica. Se entiende esta última como aquellos aspectos relacionales entre terapeuta y paciente asociados con el deseo consciente o no de este último de cooperar con el profesional que está haciendo su mayor esfuerzo para que exista un mutuo acuerdo de trabajar juntos hacia objetivos fijados en función del motivo de consulta.

Se analizó el caso de una paciente adolescente y su terapeuta, en el que ambas lograron construir y experimentar un tipo particular de relación que, en ocasiones, facilitó el trabajo tendiente al cambio terapéutico de la paciente, pero, que en otros momentos, también lo obstaculizó, debido a tensiones temporales en el vínculo. Hay que mencionar que la terapeuta fue capaz, la mayor parte de las veces, de 
identificar estos momentos de desacuerdo-no-colaborativo que terminaban muchas veces movilizando mecanismos defensivos de la paciente para mantener su equilibrio interno. Pero también fue capaz de elaborar dichas dificultades junto con la paciente, con tal de mantener intacto el vínculo y procurar el éxito de la terapia (Everall \& Paulson, 2002). Ambas fueron construyendo juntas una experiencia relacional positiva durante las primeras sesiones, que no solo les permitió trabajar colaborativamente las metas y tareas propias de la terapia durante esta primera fase del proceso, sino que además tuvieron la posibilidad de desarrollar juntas una relación afectiva, lo suficientemente estable como para resolver los momentos de ruptura, que empezaron a aparecer durante la segunda fase de la terapia en adelante. Esto coincide con la necesidad de confianza mutua, agrado, respeto y comprensión desde las primeras sesiones (Horvath \& Bedi, 2002; Philips, Wennberg \& Werbart, 2007).

Daniela llegó con una sintomatología depresiva grave, que la ubicaba dentro de la población disfuncional. No obstante, a medida que el proceso avanzaba, empezó a percibirse a sí misma cada vez menos sintomática y más funcional. Si bien es cierto que este cambio se debe en parte al tratamiento farmacológico, también pudo haber estado relacionado con el desarrollo de una alianza terapéutica temprana (Clark, 2013; Kool, Dekker, Duijsens, de Jonghe \& Puite, 2003; Labouliere, Reyes, Shirk \& Karver, 2017; Shirk, Gudmundsen, Crisp Kaplinski \& McMakin, 2008). Hay que recordar que Daniela cumplía con los criterios necesarios para ser diagnosticada con una difusión de identidad, lo que complejizaba más el caso, ya que son conocidos sus efectos adversos en la recuperación de la depresión, en términos de mejoría y remisión de síntomas, cuando se presentan conjuntamente con un trastorno de personalidad (Grilo et al., 2005; Mulder, 2002; Reich, 2003; Shea et al., 2004). Daniela frecuentemente "actuó" [mostró] durante la sesión patrones relacionales disfuncionales, intensos e inestables (Waldinger \& Gunderson, 1984), los cuales son similares a los mostrados por los pacientes con un trastorno límite de la personalidad. Frente a esto, es necesario poder construir una adecuada alianza terapéutica, justamente porque estos pacientes suelen presentar dificultades severas para establecer y mantener una adecuada alianza terapéutica (Hilsenroth, Defife, Blake \& Cromer, 2007), mostrándose más sensibles a los impases en las relaciones sociales. Por lo tanto, la resolución de rupturas se convierte en un punto clave para la obtención de resultados terapéuticos positivos (Bennett et al., 2006), sobre todo cuando reducen la probabilidad de abandonar el tratamiento (Muran, Safran, Samstag \& Winston, 2005; Strauss et al., 2006).

En el caso de Daniela, los momentos de ruptura lograron activar inmediatamente en la terapeuta la necesidad de explorar y reflexionar sobre sus patrones relacionales, con el fin de desarrollar gradualmente formas más adaptativas y flexibles de negociar las demandas y ofertas relacionales (Safran \& Muran, 2000). Terapéuticamente hablando, Daniela tuvo la posibilidad de revisar sus patrones interpersonales dentro del espacio terapéutico, lo que posibilitó que fuera adquiriendo experiencias relacionales diferentes a las que hasta entonces había conocido y, en esta medida, poder ampliar su repertorio relacional y reflexionar sobre las consecuencias de sus acciones sobre los otros. La suma de momentos de encuentro y desencuentro con su terapeuta permitió a Daniela ir transformando su sí mismo y poder establecer relaciones más sanas en el espacio extraterapéutico. En otras palabras, pudo poner en práctica en su vida cotidiana la capacidad de negociar sus necesidades relacionales y corregir los patrones generadores de conflicto en sus relaciones interpersonales (Ackerman \& Hilsenroth, 2001; Safran \& Kraus, 2014), siendo estas situaciones cotidianas del adolescente las que evocan decisiones diarias que terminan definiendo su sí mismo.

Si bien es cierto que las rupturas de la alianza terapéutica pueden llevar a resultados negativos de la terapia, los resultados de algunas investigaciones han mostrado que las relaciones terapéuticas que experimentan disminuciones de la alianza, seguida por un aumento de la misma, pueden tener el mismo o mejor resultado terapéutico que las relaciones terapéuticas en las cuales el vínculo se ha mantenido estable o en progresivo aumento a lo largo de la terapia (Kivlighan Jr. \& Shaughnessy, 2000; Stiles et al., 2004). En este sentido, los momentos de tensión en la alianza terapéutica podrían ser considerados como oportunidades que terminan facilitando mayores y mejores cambios a lo largo de la terapia, sobre todo en las terapias de modalidad individual (Safran et al., 2002). Es esperable que los pacientes experimenten sentimientos negativos con respecto a la terapia y al terapeuta, lo que exige estar atentos a la aparición de los mismos durante la sesión, a fin de alentarlos a expresar explícitamente dichos sentimientos y poder, así, explorar de manera no defensiva y colaborativamente las razones que pudieron haber causado el momento de tensión.

También se observó cómo Daniela antes del tratamiento presentaba estrategias muy pobres para la resolución de problemas. Era esperable que, ante la presencia de síntomas depresivos, la paciente reportara un estilo orientado negativamente hacia los problemas y su estilo para la solución de los mismos fuera impulsivo y evitativo (Kramp, 2012; Shaheen \& Alam, 2010; Vijayabanu \& Philip, 2013). Sin embargo, al 
finalizar la terapia, pudo desarrollar la capacidad de desplegar estrategias más adaptativas para resolver conflictos satisfactoriamente, siendo este otro indicador exitoso del resultado terapéutico obtenido. Se podría pensar que la adquisición de estas estrategias a lo largo del proceso terapéutico guarda relación con la posibilidad que tuvo la paciente durante los momentos de ruptura de actuar con su terapeuta, habilidades correctivas que le permitieran enfrentar mejor sus distintas experiencias emocionales, dentro y fuera de la sesión (Aspland, Llewelyn, Hardy, Barkham \& Stiles, 2008; Leiper \& Kent, 2001; Waddington, 2002; Watson \& Greenberg, 2000). Esto, de alguna forma, calza con los cambios observados en Daniela a nivel sintomático, puesto que la habilidad para resolver problemas sociales tendría un rol moderador y/o reductor de los efectos negativos del estrés, en presencia de un trastorno depresivo (Kant, D'Zurilla \& Maydeu-Olivares, 1997).

Algunos estudios consideran la figura del terapeuta como uno de los factores más importantes cuando se trabaja con pacientes adolescentes (Hutterer \& Liss, 2006). En este sentido, el rol de la terapeuta a nivel relacional fue crucial, en tanto se mostró presente, curiosa e interesada por la experiencia del self de la paciente, vale decir, su lenguaje corporal, su tono afectivo y un discurso consistente dirigido en todo momento a entender la experiencia relacional con su paciente en el aquí y ahora. Pudo modelarle distintas formas de involucramiento interpersonal, que le permitieran clarificar su experiencia emocional y aprender a negociar las diferencias relacionales de una mejor manera (Newirth, 2000). Tanto los intentos de resolución como la resolución exitosa de las rupturas posibilitaron que la paciente pudiera elaborar durante la sesión aquellos sentimientos y recuerdos experienciados de manera evitativa (Bennett et al., 2006; Clarkin, Yeomans \& Kernberg, 2006).

De acuerdo a Safran y Muran (2000), existen formas directas e indirectas para intentar resolver estos momentos de tensión en el vínculo. En este caso, se observó que la terapeuta generalmente respondía describiendo los fundamentos de las intervenciones terapéuticas, clarificando malentendidos relacionados con la interacción y dedicando tiempo a la exploración de los contenidos interpersonales que podían estar contribuyendo a la generación de la tensión en el vínculo, especialmente cuando el modelo exige prestar atención a la transferencia de las relaciones objetales internas del paciente durante la sesión, además de entender la contratransferencia como la capacidad de desarrollar una imagen afectiva positiva del paciente, reflejada en una actitud optimista que le permita verse a sí mismo de distintas formas (Foelsch et al., 2014).

Las investigaciones muestran que un elemento importante para la construcción de una alianza positiva es la capacidad del terapeuta para mostrar empatía (Liddle et al., 2001). Sin embargo, también se ha observado que, cuando no se involucra al adolescente en aquello que ocurre durante la sesión, también se termina afectando negativamente el vínculo y la colaboración entre ambos. En este caso, fueron evidentes las acciones realizadas por la terapeuta para focalizar la atención de ambas en las causas de la ruptura. Cuando Daniela era capaz de expresar sus sentimientos negativos, la terapeuta solía mostrar empatía como una manera de solidarizar, o bien, reconocer su parte de responsabilidad en los hechos (Safran \& Muran, 2000/2005). En cambio, cuando Daniela no era capaz de expresar sus sentimientos negativos adecuadamente, la respuesta de la terapeuta consistía en explorar las emociones asociadas con dicha tensión. La manera más frecuentemente de hacerlo era utilizando preguntas directas dicotómicas (e.g., "¿qué sientes Daniela cuando te digo esto?"), o bien, utilizar afirmaciones tendientes a reflejar ciertos afectos, ideas o conductas mostradas por la paciente (e.g., "estás enojada porque lo noto en tu cara y porque estás callada y no piensas hablar más"). Además, resulta interesante que en casi todos los momentos de ruptura estas acciones fueron utilizadas por la terapeuta para proveer experiencias inmediatas que terminaran posibilitando cambios de perspectiva en Daniela, asociados con la integración de aspectos positivos y negativos de sí misma.

\section{Conclusiones}

A partir de los resultados obtenidos, se confirma que la alianza terapéutica es más que simplemente un factor no específico que permite predecir el éxito terapéutico. La identificación de ciertos marcadores verbales y no verbales de ruptura de la alianza terapéutica durante la sesión, así como la resolución conjunta de las mismas, es un tema de investigación novedoso en el campo de la terapia con adolescentes, pero que, además, permite dar cuenta de lo complejo y multidimensional que resulta la interacción de estos marcadores con el proceso de cambio del paciente. Estos resultados contribuyen a una mayor comprensión de los altos y bajos que sufre el proceso de desarrollo de la alianza terapéutica a lo largo del tiempo. Esta metodología de análisis puede ser considerada para la formación y supervisión de los terapeutas, justamente por la riqueza clínica que se obtiene a partir de la habilidad de observar las sesiones de manera rigurosa y sistemática. Esta habilidad puede ser desarrollada con el propósito de utilizarla como un recurso que permita monitorear 
aquello que ocurre dentro de la sesión y que facilita u obstaculiza el desarrollo de una adecuada alianza terapéutica.

En el trabajo realizado con la paciente, las tensiones en el vínculo fueron una oportunidad para el cambio terapéutico, ya que cada momento de tensión entre la paciente y su terapeuta permitió, en cierta forma, ir transformando la relación de ambas, lo que, a su vez, tuvo un impacto en el proceso de cambio. Era fácil suponer que, para resolver los problemas de la alianza, iba a ser necesario enfocarse en la experiencia afectiva; sin embargo, este tipo de intervención pareciera ser mucho más importante con los pacientes diagnosticados con un trastorno de personalidad borderline, debido a la imposibilidad de autorregularse emocionalmente en sus relaciones interpersonales. Las viñetas empleadas para ejemplificar los clusters de rupturas y resoluciones reflejan las diferentes formas en que la paciente enfrentó la tensión entre la necesidad de individualizarse y relacionarse con otros.

Haber analizado todas las sesiones, y no únicamente aquellas en las que paciente y terapeuta reportan problemas en la alianza, puede ser considerado una ventaja. Analizar todas las sesiones momento a momento permitió que no quedaran fuera de la muestra sesiones con momentos de ruptura. Además, se analizaron las conductas no verbales de la paciente durante estos momentos relevantes, lo que ayudaba, muchas veces, a confirmar o descartar una tensión en el vínculo. Evidentemente, no es posible concluir que el cambio en la paciente se deba exclusivamente al trabajo realizado durante las sesiones individuales, ya que paralelamente se realizaron reuniones periódicas con los padres para apoyar el trabajo realizado individualmente, entregándoles información sobre las tareas del desarrollo normal, la naturaleza de la patología, las dificultades asociadas y optimizar el compromiso de la familia con el tratamiento.

Entre las limitaciones de este estudio está el hecho de haber considerado un enfoque de tratamiento específico, por lo que sería conveniente considerar otros enfoques, de manera de identificar posibles diferencias como consecuencia de los mismos, sobre todo, porque el modelo AIT tiene entre sus principales focos prestar especial atención a las relaciones interpersonales del paciente, dentro y fuera de la sesión, vale decir, con el terapeuta y con otros significativos, respectivamente. Sin embargo, se proyecta replicar este estudio, incluyendo en la muestra casos no exitosos que permitan contrastar los resultados exitosos obtenidos en este caso. También se espera incluir la percepción de paciente y terapeuta a lo largo del proceso, como una forma de confirmar y complementar la mirada de los observadores expertos externos. Además, se espera profundizar en temas más específicos, tales como la manera en que interactúa la resolución de las rupturas con los momentos de cambio del paciente, la manera en que son utilizadas las principales técnicas del modelo AIT (clarificar, confrontar e interpretar) durante los momentos de ruptura de la alianza, y cómo facilitan o no la resolución de las mismas, y la relación entre los momentos de ruptura y los procesos de transferencia y contratransferencia.

\section{Referencias}

Ackerman, S. J. \& Hilsenroth, M. J. (2001). A review of therapist characteristics and techniques negatively impacting the therapeutic alliance. Psychotherapy, 38, 171-185. https://doi.org/10.1037/0033-3204.38.2.171

Aspland, H., Llewelyn, S., Hardy, G. E., Barkham, M. \& Stiles,W. (2008). Alliance ruptures and rupture resolution in cognitive-behavior therapy: A preliminary task analysis. Psychotherapy Research, 18, 699-710. https://doi.org/10.1080/10503300802291463

Bachelor, A. (2013). Clients' and therapists' views of the therapeutic alliance: Similarities, differences and relationship to therapy outcome. Clinical Psychology \& Psychotherapy, 20, 118-135. https://doi.org/10.1002/cpp.792

Badenoch, B. (2008). Being a brain-wise therapist: A practical guide to interpersonal neurobiology. New York, NY: Norton.

Baillargeon, P., Coté, R. \& Douville, L. (2012). Resolution process of therapeutic alliance ruptures: A review of the literature. Psychology, 3, 1049-1058. https://doi.org/10.4236/psych.2012.312156

Bambling, M., King, R., Raue, P., Schweitzer, R. \& Lambert, W. (2006). Clinical supervision: Its influence on client-rated working alliance and client symptom reduction in the brief treatment of major depression. Psychotherapy Research, 16, 317-331. https://doi.org/10.1080/10503300500268524

Baylis, P. J., Collins, D. \& Coleman, H. (2011). Child alliance process theory: A qualitative study of a child centreed therapeutic alliance. Child \& Adolescent Social Work Journal, 28, 79-95. https://doi.org/10.1007/s10560-011-0224-2

Beck, A. T., Ward, C. H., Mendelson, M., Mock, J. \& Erbaugh, J. (1961). An inventory for measuring depression. Archives of General Psychiatry, 4, 561-571. https://doi.org/10.1001/archpsyc.1961.01710120031004

Bennett, D., Parry, G. \& Ryle, A. (2006). Resolving threats to the therapeutic alliance in cognitive analytic therapy of borderline personality disorder: A task analysis. Psychology and Psychotherapy: Theory, Research and Practice, 79, 395-418. https://doi.org/10.1348/147608305X58355

Bhola, P. \& Kapur, M. (2013). The development and role of the therapeutic alliance in supportive psychotherapy with adolescents. Psychological Studies, 58, 207-215. https://doi.org/10.1007/s12646-013-0191-0

Binder, P. -E., Holgersen, H. \& Nielsen, G. H. (2008). Re-establishing contact: A qualitative exploration of how therapists work with alliance ruptures in adolescent psychotherapy. Counselling \& Psychotherapy Research, 8, 239-245. https://doi.org/10.1080/14733140802363167 
Bolton Oetzel, K. \& Scherer, D. G. (2003). Therapeutic engagement with adolescents in psychotherapy. Psychotherapy, 40, 215-225. https://doi.org/10.1037/0033-3204.40. 3.215

Bordin, E. S. (1979). The generalizability of the psychoanalytic concept of the working alliance. Psychotherapy, 16, 252-260. https://doi.org/10.1037/h0085885

Burlingame, G. M., Cox, J. C., Wells, M. G., Lambert, M. J., Latkowski, M. \& Ferre, R. (2005). Administration and scoring manual for the Y-OQ (Youth Outcome Questionnaire). Salt Lake City, UT: American Professional Credentialing Services.

Calero, M. D., Luna, M. J., Vera-Villarroel, P. E. \& González, M. C. (2001). Un estudio de validez del Inventario de Solución de Problemas Sociales. Psicología Conductual, 9, 373-387. Extraído de https://dialnet.unirioja.es/servlet/articulo?codigo=2826483

Cecero, J. J., Fenton, L. R., Frankforter, T. L., Nich, C. \& Carroll, K. M. (2001). Focus on therapeutic alliance: The psychometric properties of six measures across three treatments. Psychotherapy, 38, 1-11. https://doi.org/10.1037/0033-3204.38.1.1

Clark, C. M. (2013). Irreducibly human encounters: Therapeutic alliance and treatment outcome in child and adolescent psychotherapy. Journal of Infant, Child, and Adolescent Psychotherapy, 12, 228-243. https://doi.org/10.1080/15289168.2013.822751

Clarkin, J. F., Yeomans, F. E. \& Kernberg, O. F. (2006). Psychotherapy for borderline personality disorder: Focusing on object relations. Washington, DC: American Psychiatric Publishing.

Constantino, M. J., Castonguay, L. G., Zack, S. E. \& DeGeorge, J. (2010). Engagement in psychotherapy: Factors contributing to the facilitation, demise, and restoration of the therapeutic alliance. En D. Castro-Blanco \& M. S. Karver (Eds.), Elusive alliance: Treatment engagement strategies with high-risk adolescents (pp. 199-209). Washington, DC: American Psychological Association.

Daly, A. -M., Llewelyn, S., McDougall, E. \& Chanen, A. M. (2010). Rupture resolution in cognitive analytic therapy for adolescents with borderline personality disorder. Psychology and Psychotherapy: Theory, Research and Practice, 83, 273-288. https://doi.org/10.1348/147608309X481036

Diamond, G., Siqueland, L. \& Diamond, G. M. (2003). Attachment-based family therapy for depressed adolescents: Programmatic treatment development. Clinical Child and Family Psychology Review, 6, 107-127. https://doi.org/10.1023/A:1023782510786

Duncan, B. L., Miller, S. D., Wampold, B. E. \& Hubble, M. A. (2010). The heart and soul of change: Delivering what works in therapy ( $2^{a}$ ed.). Washington, DC: American Psychological Association.

D'Zurilla, T. J., Nezu, A. M. \& Maydeu-Olivares, A. (1999). Manual for the Social Problem-Solving Inventory-Revised. North Tonawanda, NY: Multi-Health Systems.

Escudero, V., Boogmans, E., Loots, G. \& Friedlander, M. L. (2012). Alliance rupture and repair in conjoint family therapy: An exploratory study. Psychotherapy, 49, 26-37. https://doi.org/10.1037/a0026747

Eubanks, C. F., Muran, J. C. \& Safran, J. D. (2015). Rupture Resolution Rating System (3RS): Manual. New York, NY: Mount SinaiBeth Israel Medical Center.

Everall, R. D. \& Paulson, B. L. (2002). The therapeutic alliance: Adolescent perspectives. Counselling \& Psychotherapy Research, 2 , 78-87. https://doi.org/10.1080/14733140212331384857

Eyrich-Garg, K. M. (2008). Strategies for engaging adolescent girls at an emergency shelter in a therapeutic relationship: Recommendations from the girls themselves. Journal of Social Work Practice, 22, 375-388. https://doi.org/10.1080/02650530802396700

Faw, L., Hogue, A., Johnson, S., Diamond, G. M. \& Liddle, H. A. (2005). The Adolescent Therapeutic Alliance Scale (ATAS): Initial psychometrics and prediction of outcome in family-based substance abuse prevention counselling. Psychotherapy Research, 15, 141-154. https://doi.org/10.1080/10503300512331326994

Fenichel, O. (1936/1941). Problems of psychoanalytic technique (D. Brunswick, Trad; Título original: fue traducido de un seminario denominado "Probleme der Technik"). New York, NY: The Psychoanalytic Quarterly.

Fernández, O., Pérez, C., Gloger, S. \& Krause M. (2015). Importancia de los cambios iniciales en la psicoterapia con adolescentes. Terapia Psicológica, 33, 247-255. https://doi.org/10.4067/S0718-48082015000300008

Fitzpatrick, M. R. \& Irannejad, S. (2008). Adolescent readiness for change and the working alliance in counseling. Journal of Counseling \& Development, 86, 438-445. https://doi.org/10.1002/j.1556-6678.2008.tb00532.x

Foelsch, P. A., Schlüter-Müller, S., Odom, A. E., Arena, H. T., Borzutzky, A. \& Schmeck, K. (2014). Adolescent identity treatment: An integrative approach for personality pathology. Basel, Suiza: Springer.

Freud, S. (1912/1958). The dynamics of transference (J. Riviere, Trad.; Título original: Zur Dynamik der Übertragung). En J. Strachey (Ed.) The standard edition of the complete psychological works of Sigmund Freud, Volume XII (1911-1913): The case of Schreber, papers on technique and other works (pp. 97-108). London, Reino Unido: Hogarth Press.

Friedlander, M. L., Kivlighan Jr., D. M. \& Shaffer, K. S. (2012). Exploring actor-partner interdependence in family therapy: Whose view (parent or adolescent) best predicts treatment progress? Journal of Counseling Psychology, 59, 168-175. https://doi.org/10.1037/a0024199

Goth, K., Foelsch, P., Schlüter-Müller, S., Birkhölzer, M., Jung, E., Pick, O. \& Schmeck, K. (2012). Assessment of identity development and identity diffusion in adolescence - theoretical basis and psychometric properties of the self-report questionnaire AIDA. Child and Adolescent Psychiatry and Mental Health, 6, artículo 27. https://doi.org/10.1186/1753-2000-6-27

Green, J. (2006). Annotation: The therapeutic alliance - a significant but neglected variable in child mental health treatment studies. Journal of Child Psychology and Psychiatry, 47, 425-435. https://doi.org/10.1111/j.1469-7610.2005.01516.x

Greenberg, L. S. (1984). Task analysis: The general approach. En L. N. Rice \& L. S. Greenberg (Eds.), Patterns of change: Intensive analysis of psychotherapy process (pp. 124-148). New York, NY: Guilford Press.

Greenson, R. R. (1965). The working alliance and the transference neurosis. The Psychoanalytic Quarterly, 34, 155-181. Extraído de https://www.ncbi.nlm.nih.gov/pubmed/14302976

Grilo, C. M., Sanislow, C. A., Shea, M. T., Skodol, A. E., Stout, R. L., Gunderson, J. G. ... McGlashan, T. H. (2005). Two-year prospective naturalistic study of remission from major depressive disorder as a function of personality disorder comorbidity. Journal of Consulting and Clinical Psychology, 73, 78-85. https://doi.org/10.1037/0022-006X.73.1.78

Hill, C. E., Knox, S., Thompson, B. J., Williams, E. N., Hess, S. A. \& Ladany, N. (2005). Consensual qualitative research: An update. Journal of Counseling Psychology, 52, 196-205. https://doi.org/10.1037/0022-0167.52.2.196

Hilsenroth, M. J., Defife, J. A., Blake, M. M. \& Cromer, T. D. (2007). The effects of borderline pathology on short-term psycodynamic psychotherapy for drepression. Psychotherapy Research, 17, 175-184. https://doi.org/10.1080/10503300600786748

Hogue, A., Daubter, S., Stambaugh, L. F., Cecero, J. J. \& Liddle, H. A. (2006). Early therapeutic alliance and treatment outcome in individual and family therapy for adolescent behavior problems. Journal of Consulting and Clinical Psychology, 74, 121-129. https://doi.org/10.1037/0022-006X.74.1.121 
Horvath, A. O. (2006). The alliance in context: Accomplishments, challenges, and future directions. Psychotherapy, 43, 258-263. https://doi.org/10.1037/00333204.43.3.258

Horvath, A. O. (2009). How real is the "real relationship"? Psychotherapy Research, 19, 273-277. https://doi.org/10.1080/10503300802592506

Horvath, A. O. \& Bedi, R. P. (2002). The alliance. En J. C. Norcross (Ed.), Psychotherapy relationships that work: Evidence-based responsiveness (pp. 37-69). New York, NY: Oxford University Press.

Horvath, A. O., Del Re, A. C., Flückiger, C. \& Symonds, D. (2011). Alliance in individual psychotherapy. Psychotherapy, 48, 9-16. https://doi.org/10.1037/a0022186

Hunter, J. A., Button, M. L. \& Westra, H. A. (2014). Ambivalence and alliance ruptures in cognitive behavioral therapy for generalized anxiety. Cognitive Behaviour Therapy, 43, 201-208. https://doi.org/10.1080/16506073.2014.899617

Hutterer, J. \& Liss, M. (2006). Cognitive development, memory, trauma, treatment: An integration of psychoanalytic and behavioral concepts in light of current neuroscience research. Psychodynamic Psychiatry, 34, 287-302. https://doi.org/10.1521/jaap.2006.34.2.287

Kant, G. L., D'Zurilla, T. J. \& Maydeu-Olivares, A. (1997). Social problem solving as a mediator of stress-related depression and anxiety in middle-aged and elderly community residents. Cognitive Therapy and Research, 21, 73-96. https://doi.org/10.1023/A:1021820326754

Karver, M. S., Handelsman, J. B., Fields, S. \& Bickman, L. (2006). Meta-analysis of therapeutic relationship variables in youth and family therapy: The evidence for different relationship variables in the child and adolescent treatment outcome literature. Clinical Psychology Review, 26, 50-65. https://doi.org/10.1016/j.cpr.2005.09.001

Kazdin, A. E. (2008). Evidence-based treatment and practice: New opportunities to bridge clinical research and practice, enhance the knowledge base, and improve partient care. American Psychologist, 63, 146-159. https://doi.org/10.1037/003-066x.63.3.146

Kazdin, A. E. \& Whitley, M. K. (2006). Pretreatment social relations, therapeutic alliance, and improvements in parenting practices in parent management training. Journal of Consulting and Clinical Psychology, 74, 346-355. https://doi.org/10.1037/0022006X.74.2.346

Kivlighan Jr., D. M. \& Shaughnessy, P. (2000). Patterns of working alliance development: A typology of client's working alliance ratings. Journal of Counseling Psychology, 47, 362-371. https://doi.org/10.1037/0022-0167.47.3.362

Kool, S., Dekker, J., Duijsens, I. J., de Jonghe, F. \& Puite, B. (2003). Efficacy of combined therapy and pharmacotherapy for depressed patients with or without personality disorders. Harvard Review of Psychiatry, 11, 133-141. https://doi.org/10.1080/10673220303950

Kramp, U. (2012). Perfil de resolución de problemas sociales y afrontamiento en ansiosos y depresivos chilenos. Psicothema, 24, 529-535. Extraído de http://www.psicothema.com/pdf/4049.pdf

Labouliere, C. D., Reyes, J. P., Shirk, S. \& Karver, M. (2017). Therapeutic alliance with depressed adolescents: Predictor or outcome? Disentangling temporal confounds to understand early improvement. Journal of Clinical Child \& Adolescent Psychology, 46, 600-610. https://doi.org/10.1080/15374416.2015.1041594

Leiper, R. \& Kent, R. (2001). Working through setbacks in psychotherapy: Crisis, impasse and relapse. London, Reino Unido: SAGE. https://doi.org/10.4135/9781446217344

Liber, J. M., McLeod, B. D., Van Widenfelt, B. M., Goedhart, A. W., van der Leeden, A. J. M., Utens, E. M. W. J. \& Treffers, P. D. A. (2010). Examining the relation between the therapeutic alliance, treatment adherence, and outcome of cognitive behavioral therapy for children with anxiety disorders. Behavior Therapy, 41, 172-186. https://doi.org/10.1016/j.beth.2009.02.003

Liddle, H. A., Dakof, G. A., Parker, K., Diamond, G. S., Barrett, K. \& Tejeda, M. (2001). Multidimensional family therapy for adolescent drug abuse: Results of a randomized clinical trial. The American Journal of Drug and Alcohol Abuse, 27, 651-688. https://doi.org/10.1081/ADA100107661

Lindsey, M. A., Brandt, N. E., Becker, K. D., Lee, B. R., Barth, R. P., Daleiden, E. L. \& Chorpita, B. F. (2014). Identifying the common elements of treatment engagement interventions in children's mental health services. Clinical Child and Family Psychology Review, 17, 283-298. https://doi.org/10.1007/s10567-013-0163-x

Lingiardi, V., Gazzillo, F. \& Waldron Jr., S. (2010). An empirically supported psychoanalysis: The case of Giovanna. Psychoanalytic Psychology, 27, 190-218. https://doi.org/10.1037/a0019418

Luborsky, L. (1976). Helping alliances in psychotherapy. En J. L. Cleghorn (Ed.), Successful psychotherapy (pp. 92-116). New York, NY: Brunner/Mazel.

Malan, D. H. (2007). Individual psychotherapy and the science of psychodynamics (2a ed.). London, Reino Unido: Hodder Education.

Martin, D. G. (2003). Clinical practice with adolescents. Pacific Grove, CA: Brooks/Cole-Thomson Learning.

Martin, D. J., Garske, J. P. \& Davis, M. K. (2000). Relation of the therapeutic alliance with outcome and other variables: A meta-analysis review. Journal of Consulting and Clinical Psychology, 68, 438-450. https://doi.org/10.1037/0022-006X.68.3.438

McLeod, B. D. \& Weisz, J. R. (2005). The Therapy Process Observational Coding System-Alliance Scale: Measure characteristics and prediction of outcome in usual clinical practice. Journal of Consulting and Clinical Psychology, 73, 323-333. https://doi.org/10.1037/0022-006X.73.2.323

Mulder, R. T. (2002). Personality pathology and treatment outcome in major depression: A review. The American Journal of Psychiatry, 159, 359-371. https://doi.org/10.1176/appi.ajp.159.3.359

Muran, J. C., Safran, J. D., Samstag, L. W. \& Winston, A. (2005). Evaluating an alliance-focused treatment for personality disorders. Psychotherapy, 42, 532-545. https://doi.org/10.1037/0033-3204.42.4.532

Newirth, J. (2000). Impasses in the psychoanalytic relationship. Journal of Clinical Psychology, 56, 225-231. https://doi.org/10.1002/(SICI)10974679(200002)56:2<225::AID-JCLP8>3.0.CO;2-7

Pachankis, J. E. \& Goldfried, M. R. (2007). On the next generation of process research. Clinical Psychology Review, 27, 760-768. https://doi.org/10.1016/j.cpr.2007.01.009

Philips, B., Wennberg, P. \& Werbart, A. (2007). Ideas of cure as a predictor of premature termination, early alliance and outcome in psychoanalytic psychotherapy. Psychology and Psychotherapy: Theory, Research and Practice, 80, $229-245$. https://doi.org/10.1348/147608306X128266

Platt, D. (2008). Care or control? The effects of investigations and initial assessments on the social worker-parent relationship. Journal of Social Work Practice, 22, 301-315. https://doi.org/10.1080/02650530802396643

Pos, A. E., Greenberg, L. S. \& Warwar, S. H. (2009). Testing a model of change in the experiential treatment of depression. Journal of Consulting and Clinical Psychology, 77, 1055-1066. https://doi.org/10.1037/a0017059

Reich, J. (2003). The effect of axis II disorders on the outcome of treatment of anxiety and unipolar depressive disorders: A review. Journal of Personality Disorders, 17, 387-405. https://doi.org/10.1521/pedi.17.5.387.22972 
Safran, J. D. \& Kraus, J. (2014). Alliance ruptures, impasses, and enactments: A relational perspective. Psychotherapy, 51, 381-387. https://doi.org/10.1037/a0036815

Safran, J. D. \& Muran, J. C. (2000). Resolving therapeutic alliance ruptures: Diversity and integration. Journal of Clinical Psychology, 56, 233-243. https://doi.org/10.1002/(SICI)1097-4679(200002)56:2<233::AID-JCLP9>3.0.CO;2-3

Safran, J. D. \& Muran, J. C. (2000/2005). La alianza terapéutica: una guía para el tratamiento relacional (Título original: Negotiation the therapeutic aliance: A relational treatment guide). Bilbao, España: Desclée de Brouwer.

Safran, J. D., Muran, J. C. \& Eubanks-Carter, C. (2011). Repairing alliance ruptures. Psychotherapy, 48, 80-87. https://doi.org/10.1037/a0022140 Safran, J. D., Muran, J. C., Samstag, L. W. \& Stevens, C. (2002). Repairing alliance ruptures. En J. C. Norcross (Ed.), Psychotherapy relationships that work: Therapist contributions and responsiveness to patient needs (pp. 235-254). New York, NY: Oxford University Press.

Sameroff, A. (Ed.) (2009). The transactional model of development: How children and contexts shape each other. Washington, DC: American Psychological Association.

Santibáñez Fernández, P. M., Román Mella, M. F. \& Vinet, E. V. (2009). Efectividad de la psicoterapia y su relación con la alianza terapéutica. Interdisciplinaria, 26, 267-287. Extraído de http://www.redalyc.org/pdf/180/18011827006.pdf

Shaheen, F. \& Alam, S. (2010). Psychological distress and its relation to attributional styles and coping strategies among adolescents. Journal of the Indian Academy of Applied Psychology, 36, 231-238. Extraído de http://medind.nic.in/jak/t10/i2/jakt10i2p231.pdf

Shea, M. T., Stout, R. L., Yen, S., Pagano, M. E., Skodol, A. E., Morey, L. C. ... Zanarini, M. C. (2004). Associations in the course of personality disorders and axis I disorders over time. Journal of Abnormal Psychology, 113, 499-508. https://doi.org/10.1037/0021843x.113.4.499

Shelef, K., Diamond, G. M., Diamond, G. S. \& Liddle, H. A. (2005). Adolescent and parent alliance and treatment outcome in multidimensional family therapy. Journal of Consulting and Clinical Psychology, 73, 689-698. https://doi.org/10.1037/0022006x.73.4.689

Shirk, S. R., Gudmundsen, G., Crisp Kaplinski, H. C. \& McMakin, D. L. (2008). Alliance and outcome in cognitive-behavioral therapy for adolescent depression. Journal of Clinical Child \& Adolescent Psychology, 37, 631-639. https://doi.org/10.1080/15374410802148061

Shirk, S. R. \& Karver, M. S. (2011). Alliance in child and adolescent psychotherapy. En J. C. Norcross (Ed.), Psychotherapy relationships that work: Evidence-based responsiveness (2a ed., pp. 70-91). New York, NY: Oxford University Press.

Staudt, M. (2007). Treatment engagement with caregivers of at-risk children: Gaps in research and conceptualization. Journal of Child and Family Studies, 16, 183-196. https://doi.org/10.1007/s10826-006-9077-2

Stiles, W. B., Glick, M. J., Osatuke, K., Hardy, G. E., Shapiro, D. A., Agnew-Davies, R. ... Barkham, M. (2004). Patterns of alliance development and the rupture-repair hypothesis: Are productive relationships U-shaped or V-shaped? Journal of Counseling Psychology, 51, 81-92. https://doi.org/10.1037/0022-0167.51.1.81

Strauss, J. L., Hayes, A. M., Johnson, S. L., Newman, C. F., Brown, G. K., Barber, J. P. ... Beck, A. T. (2006). Early alliance, alliance ruptures, and symptom change in a nonrandomized trial of cognitive therapy for avoidant and obsessive-compulsive personality disorders. Journal of Consulting and Clinical Psychology, 74, 337-345. https://doi.org/10.1037/0022-006x.74.2.337

Strupp, H. H. (1973). On the basic ingredients of psychotherapy. Journal of Consulting and Clinical Psychology, 41, 1-8. https://doi.org/10.1037/h0035619

Sylwester, R. (2007). The adolescent brain: Reaching for autonomy. Thousand Oaks, CA: Corwin Press.

Tryon, G. S., Blackwell, S. C. \& Hammel, E. F. (2007). A meta-analytic examination of client-therapist perspectives of the working alliance. Psychotherapy Research, 17, 629-642. https://doi.org/ 10.1080/10503300701320611

Valdés, C., Morales-Reyes, I., Pérez, J. C., Medellín, A., Rojas, G. \& Krause, M. (2017). Propiedades psicométricas del Inventario de Depresión de Beck IA para la población chilena. Revista Médica de Chile, 145, 1005-1012. https://doi.org/10.4067/s003498872017000801005

Valdés, N. \& Hernández, C. (2017). Adaptación y validación de la versión chilena del Cuestionario de Resultado Terapéutico para Adolescentes (Y-OQ-SR 2.0). Manuscrito en preparación.

Valdés, N., Hernández, C., Goth, K., Quevedo, Y. \& Borzutzky, A. (2018). Adaptación y validación de la versión chilena del Cuestionario para Evaluar el Desarrollo de la Identidad en Adolescentes (AIDA). Manuscrito sometido para publicación.

Vijayabanu, P. U. \& Philip, J. N. (2013). Relationship of affect, resilience and social problem solving among female nursing students. Indian Journal of Positive Psychology, 4, 494-498. https://doi.org/10.15614/ijpp/2013/v4i4/49961

Vinet, E. V. \& Forns i Santacana, M. (2008). Normas chilenas para el MACI: una integración de criterios categoriales y dimensionales. Terapia Psicológica, 26, 151-163. https://doi.org/10.4067/S0718-48082008000200001

Waddington, L. (2002). The therapy relationship in cognitive therapy: A review. Behavioural and Cognitive Psychotherapy, 30, 179-191. https://doi.org/10.1017/S1352465802002059

Waldinger, R. J. \& Gunderson, J. G. (1984). Completed psychotherapies with borderline patients. American Journal of Psychotherapy, 38, 190-202. https://doi.org/10.1176/appi.psychotherapy.1984.38.2.190

Watson, J. C. \& Greenberg, L. S. (2000). Alliance ruptures and repairs in experiential therapy. Journal of Clinical Psychology, 56, 175-186. https://doi.org/10.1002/(SICI)1097-4679(200002)56:2<175::AID-JCLP4>3.0.CO;2-5

Wells, M. G., Burlingame, G. M. \& Rose, P. M. (2003). Youth Outcome Questionnaire Self-report. Wilmington, DE: American Professional Credentialing Services.

Wright, L., Everett, F. \& Roisman, L. (1986). Experiential psychotherapy with children. Baltimore, MD: Johns Hopkins University Press.

Zetzel, E. R. (1956). Current concepts of transference. The International Journal of Psychoanalysis, 37, 369-376. Extraído de https://www.ncbi.nlm.nih.gov/pubmed/13366506

\section{Anexo}

(1) "No sé, no quiero hablar de lo que pasó. O sea, para qué si ya pasó y no quiero recordar" (Sesión 13, Ventana 1, 00:00:00 - 00:04:59).

(2) “Todavía seguía bajoneada [desanimada]. Y ni siquiera me enojé, sino que me bajoneé [desanimé] más. Ellos creen que me enojo, pero la verdad es que cualquier cosa que me digan, mala o fea o hiriente, me pongo a llorar. He estado más sensible. Entonces, me fui a mi pieza a llorar y me corté en la pierna 
[muestra dónde se cortó]. Mis papás no toman en cuenta mi enojo o mi mal humor, porque creen que es una tontera, pero la verdad es que ellos no saben que he estado aguantando harto" (Sesión 15, Ventana 2, 00:05:00-00:09:59).

(3) "No es que tú no me estés ayudando, si no que no me gustas como terapeuta. No me gustan tus actitudes. Como que piensas por mí, respondes por mí, esas cosas. Perdón, pero como terapeuta no me gustas. Me caes bien, pero como terapeuta no. Me ayudaste mucho, de verdad, pero como terapeuta ya no funciona" (Sesión 31, Ventana 4, 00:15:00 - 00:19:50).

(4) "Pareciera que lo que yo digo acá no se lo dices a ellos o lo que yo pienso no se lo dices. Por ejemplo, ¿tú le vas a decir a mis papás que me molesta eso? ¿que quiero que cambien un poco? ¿se lo vas a decir? Porque yo no puedo, no me sale, me cuesta mucho hablar con ellos, no me gusta. Esto no lo voy a hacer, no me gusta" (Sesión 13, Ventana 8, 00:35:00 - 00:39:59).

(5) "No quería venir porque estoy cansada. Y también porque hoy me invitaron a la casa de un amigo y yo no podía ir, porque tenía esto [refiriéndose a la sesión]. Entonces es una lata [fastidio], porque esto me impide juntarme con personas. Me impide hacer cosas que me gustaría hacer. Por eso, es bueno que apuráramos también la cosa y venir una vez a la semana, porque es mucho" (Sesión 19, Ventana 1, 00:00:00 - 00:04:59).

(6) "Probablemente no vas a decir nada y ya sé cuándo tú no quieres hablar nada más. ¿Quieres compartir conmigo lo que estás pensando, sea lo que sea? Quizás yo tenga una visión diferente y a lo mejor tú podrías explicarme por qué yo hoy veo a una Daniela diferente a la que he visto otras veces" (Sesión 15, Ventana 4, 00:15:00 - 00:19:59).

(7) "Yo creo que hay muchas cosas que no te gustan y que evitas hacer porque no te gustan. Y tú estás esperando que yo hable por ti. Yo lo podría hacer, pero no tendría el mismo efecto. Tú has dejado de hacer muchas cosas que no te gustan y, aunque te enojes ahora conmigo, yo por ahora no me siento cómoda diciéndoles a tus papás lo que tú quieres decir, porque tú eres la que debería ser capaz de hacerlo" (Sesión 13, Ventana 8, 00:35:00 - 00:39:59).

(8) "A mí me queda dando vueltas un tema que hemos hablado por lo menos las dos últimas sesiones. Y hoy te quiero compartir lo que me pasa a mí. Yo siento que estamos como entrampadas o estancadas en esto de que ellos [padres] no saben qué es lo que te pasa a ti. Hemos estado dándole vueltas al mismo tema y siento que, como yo aún no te he dicho que sí lo voy a hacer, eso te ha generado mucha rabia conmigo" (Sesión 15, Ventana 2, 00:05:00 - 00:09:59).

(9) "A lo mejor te molesta, porque te ves reflejada a ti misma en lo que yo hago. Como cuando te muestro cuáles son los gestos o las caras que tú haces que me indican las cosas que tú no quieres decir verbalmente, pero que las dices con tu cuerpo. A lo mejor, esto te confirma cosas que te pasan con las otras relaciones, cuando te pones pesada y la gente se aleja de ti. No sé si eso es lo mismo que está pasando acá conmigo. Esto mismo te ha pasado con otras niñas, que, cuando ya te dejan de caer bien, tú las alejas y pasan a ser las que ya no son tus amigas. Quizás, entre comillas yo te dejé de caer bien y ya no quieres estar más conmigo como terapeuta" (Sesión 31, Ventana 5, 00:20:00 - 00:24:59).

(10) "Está bien, yo entiendo que no quieras recordar. Pero yo creo que no es menor lo que pasó, porque te vi muy afectada y muy enojada ... entiendo que no quieras recordar, pero creo que lo que te pasó puede que te siga pasando y, si no lo revisamos, quizás no podamos entender qué significa" (Sesión 13, Ventana 2, 00:05:00 - 00:09:59).

Fecha de recepción: Diciembre de 2016.

Fecha de aceptación: Mayo de 2018. 